\title{
Investigations on the porous resistance coefficients for fishing net structures
}

\author{
Chen, Hao; Christensen, Erik Damgaard
}

Published in:

Journal of Fluids and Structures

Link to article, DOI:

10.1016/j.jluidstructs.2016.05.005

Publication date:

2016

Document Version

Peer reviewed version

Link back to DTU Orbit

Citation (APA):

Chen, H., \& Christensen, E. D. (2016). Investigations on the porous resistance coefficients for fishing net structures. Journal of Fluids and Structures, 65, 76-107. https://doi.org/10.1016/j.jluidstructs.2016.05.005

\section{General rights}

Copyright and moral rights for the publications made accessible in the public portal are retained by the authors and/or other copyright owners and it is a condition of accessing publications that users recognise and abide by the legal requirements associated with these rights.

- Users may download and print one copy of any publication from the public portal for the purpose of private study or research.

- You may not further distribute the material or use it for any profit-making activity or commercial gain

- You may freely distribute the URL identifying the publication in the public portal

If you believe that this document breaches copyright please contact us providing details, and we will remove access to the work immediately and investigate your claim. 


\title{
Investigations on the porous resistance coefficients for fishing net structures
}

\author{
Hao Chen ${ }^{\mathrm{a}, *}$, Erik Damgaard Christensen ${ }^{\mathrm{a}}$ \\ ${ }^{a}$ Section of Fluid Mechanics, Coastal and Maritime Engineering, Department of Mechanical Engineering, Technical \\ University of Denmark, DK-2800 Kgs. Lyngby, Denmark
}

\begin{abstract}
The porous media model has been successfully applied to numerical simulation of current and wave interaction with traditional permeable coastal structures such as breakwaters. Recently this model was employed to simulate flow through and around fishing net structures, where the unknown porous resistance coefficients were adjusted by fitting the available experimental data. In the present paper, a new approach was proposed to calculate the porous resistance coefficients based on the transformation of Morison type load model. The transformation follows the principle that the total forces acting on a net panel from Morison type load model should be equal to the forces obtained from the porous media model. In order to account for the interaction effects in-between the twines, two coefficients were introduced, and they were calibrated by minimizing the least square error function. Extensive validation cases were carried out to examine the performance of the numerical model. This includes steady current flow through plane net panels and circular fish cages, and wave interaction with plane net panels. A variety of fishing nets with different solidity ratios were used in the validation cases, from which it was seen that the overall agreement between the numerical and experimental results is fair.
\end{abstract}

Keywords: porous media model, resistance coefficients, fishing nets, Navier-Stokes equations

\section{Introduction}

Porous structures are widely used in coastal engineering, e.g. breakwaters for sea defense. A number of studies have been carried out on numerical simulation of flow through and around such structures, e.g. Jensen et al. (2014), Hsu et al. (2002), Liu et al. (1999), Losada et al. (2008), del Jesus et al. (2012), Higuera et al. (2014a) and Higuera et al. (2014b). Among these works, the effect of porous structures on the fluid was taken into account without resolving the exact geometry of them. Instead, they were treated as one continuum from a macroscopic point of view. By volume averaging the Navier-Stokes equations over a representative elementary volume, the effect of porous structures was included via a resistance term. This term was usually described by the extended Darcy-Forchheimer equation, which includes linear and nonlinear forces as well as inertia forces to account for accelerations.

Recently this approach was applied to simulate flow through fishing nets. In Patursson et al. (2010), Zhao et al. (2013a) and Zhao et al. (2013b), the porous media model was applied to simulate steady flow through a single net panel with different attack angles, multiple net panels and gravity cages, respectively. Furthermore, in Bi et al. (2014a) and Bi et al. (2014b), the porous media model was coupled with a lumped mass structural model to account for fluid-structure interaction effect. An iterative scheme was set up between these two models and the steady solution of the flow field and net deformation was obtained after several iterations. Similar work was also performed in Devilliers et al. (2016), where advanced adaptive mesh refinement technique was developed to increase the mesh resolution and improve the accuracy of the numerical results. Bi et al. (2015) and Zhao et al. (2014) further applied the porous media model for wave

\footnotetext{
*Corresponding author, tel.: (45) 50302416; e-mail address: hchen@mek.dtu.dk; fax (45) 45251961.
} 


\section{Nomenclature}

$\gamma_{p} \quad$ Empirical coefficient in the expression of added mass coefficient

$\lambda \quad$ Mesh bar length of the fishing net

$\left\langle\bar{p}^{f}\right\rangle \quad$ Volume averaged ensemble averaged pore pressure

$\langle\bar{u}\rangle \quad$ Volume averaged ensemble averaged turbulent fluctuating velocity field

$\langle\bar{u}\rangle \quad$ Volume averaged ensemble averaged velocity field

$\left\langle\mu_{t}\right\rangle \quad$ Volume averaged eddy viscosity

$\langle k\rangle \quad$ Volume averaged specific turbulent kinetic energy

$\mu \quad$ Dynamic viscosity of the fluid

$\nu \quad$ Kinematic viscosity of the fluid

$\omega \quad$ Wave angular frequency

$\rho \quad$ Density of the fluid

$\theta \quad$ Attack angle, where $\theta=90^{\circ}$ indicates the flow is aligned with the normal direction of the net panel

a Interaction coefficient in the expression of normal quadratic drag resistance coefficient

$A_{n e t} \quad$ Outlined area of the net panel

$b \quad$ Interaction coefficient in the expression of tangential quadratic drag resistance coefficient

$C \quad$ Quadratic porous drag resistance coefficient

$C_{m} \quad$ Added mass coefficient of the porous media

$C_{d, n e t}$ Drag force coefficient of the net

$C_{d, t w i n e}$ Drag force coefficient of the net twines

$C_{I, t w i n e}$ Inertia force coefficient of the net twines

$C_{l, \text { net }}$ Lift force coefficient of the net

$D \quad$ Linear porous drag resistance coefficient $d \quad$ Twine diameter of the fishing nets

$F \quad$ Hydrodynamic forces acting on the porous media based on the Morison type load model

$F_{d, \text { net }}$ Drag force of the net

$F_{d, \text { twine }}$ Drag force of the net twines

$F_{I, t w i n e}$ Inertia force of the net twines

$F_{l, \text { net }} \quad$ Lift force of the net

$g \quad$ Gravitation acceleration

$K C \quad$ Keulegan-Carpenter number

$n \quad$ Porosity of the fishing net

$P \quad$ Total pressure

$p \quad$ Excess pressure, where the hydrostatic pressure is subtracted

$Q \quad$ Hydrodynamic forces acting on the porous media based on the porous media model

$R \quad$ Transformation matrix from local coordinate system to global coordinate system

Re Reynolds number

$S \quad$ Porous drag resistance

$S_{1} \quad$ Total projected area for in-plane twines of the considered net panel

$S_{2} \quad$ Total projected area for out-of-plane twines of the considered net panel

$S_{n} \quad$ Solidity ratio of the fishing net, defined as complement of porosity

$T \quad$ Wave period

$u \quad$ Velocity field

$U_{\infty} \quad$ Magnitude of the velocity at infinity for current flow

$U_{m} \quad$ Magnitude of the maximum velocity in one period for the considered wave

$V \quad$ Volume of the porous media zone

interaction with net structures. The transmission coefficients obtained from CFD simulations were compared with laboratory tests under different wave conditions.

As described above, porous media model has already been used in several papers to simulate current and wave interaction with fishing nets. The net was modeled as a sheet of porous media with very thin thickness, 
usually between $10 \mathrm{~mm}$ - $50 \mathrm{~mm}$. Unlike the model used in breakwater design, anisotropy was taken into account in porous media flow for fishing nets, due to the difference between the exerted resistances in tangential and normal directions. Darcy-Forchheimer formula was applied to calculate the porous resistance forces, where constant resistance coefficients were obtained by optimizing the fit between the measured drag and lift forces from experiments and computed drag and lift forces at different flow velocities and attack angles. This approach has advantages that it avoids direct modeling of the fishing nets geometry, which is usually unrealistic since a single net panel may have tens of thousands of twins and knots. From the validation of the numerical model provided in the above mentioned works, the predicted forces and wake velocities from the porous media model in general agreed well with laboratory experiments, which demonstrates the feasibility of this approach.

However, there remains one issue for direct application of porous media model on flow through fishing nets, i.e. finding the porous resistance coefficients in Darcy-Forchheimer equation. Fitting procedure requires that for each individual net panel, measured drag and lift forces must be available for a variety of incoming velocities and attack angles, which limits its application in practical design. The present work provides an alternative approach to calculate the porous resistance coefficients, which expresses them as a function of physical parameters of the fishing net. These parameters are easily obtained from a given net panel, therefore the resistance coefficients are directly determined without any need of experimental data. Hereby this eases the application of the numerical model.

The remainder of the paper is organized as follows. In Section 2 , a brief description is given on the numerical model, where the volume averaged Reynolds averaged Navier-Stokes equations proposed in Jensen et al. (2014) are employed as the governing equations. Section 3 gives the derivation and calibration of the expressions for porous resistance coefficients, followed by summary and discussion on the overall numerical model in Section 4. In Section 5, preliminary tests are run for the general setup and convergence analysis of the numerical model, while in Section 6 extensive validation cases are performed against laboratory tests. Series of experiments conducted in Patursson (2007), Zhan et al. (2006), Lader et al. (2007a) and Lader et al. (2007b) are reproduced by the numerical model to examine its accuracy and performance. Furthermore, sensitivity analysis is performed to test the influences of uncertainties in calculation of the porous resistance coefficients in Section 7. Finally conclusions of the paper are given in Section 8 .

\section{Description of the numerical model}

The numerical model was set up within the framework of the open source computational fluid dynamics toolbox OpenFOAM with the version of extend-3.1. It uses finite volume discretization method with a collocated variable arrangement on unstructured grid. The solvers employed in the present work solve the volume averaged Reynolds averaged Navier-Stokes (VARANS) equations. For single phase porous media flow, e.g. net panel in current, a transient single phase flow solver was employed. For two phase porous media flow where waves were involved in, the solver solves VARANS equations for two incompressible, isothermal and immiscible fluids where volume of fluid (VOF) approach (see Hirt and Nichols (1981)) was used to capture the interface.

\subsection{Governing equations}

The VARANS equations proposed in Jensen et al. (2014) are the governing equations in the present work. Start from the general form of the incompressible Navier-Stokes equations:

$$
\begin{gathered}
\frac{\partial u_{i}}{\partial x_{i}}=0 \\
\frac{\partial \rho u_{i}}{\partial t}+\frac{\partial \rho u_{i} u_{j}}{\partial x_{j}}=-\frac{\partial p}{\partial x_{i}}-g_{j} x_{j} \frac{\partial \rho}{\partial x_{i}}+\frac{\partial}{\partial x_{j}} \mu\left(\frac{\partial u_{i}}{\partial x_{j}}+\frac{\partial u_{j}}{\partial x_{i}}\right)
\end{gathered}
$$

where $x_{i}=(x, y, z)$ is the global Cartesian coordinate system. 
If free surface is involved in the numerical model for handling cases of wave propagation and interaction with fishing nets, an additional advection equation needs to be solved for local water volume fraction $\alpha$ :

$$
\frac{\partial \alpha}{\partial t}+\frac{\partial}{\partial x_{i}}\left(u_{i} \alpha\right)=0
$$

However, solving Eq. (3) introduces a number of difficulties with respect to boundness of the solution and prevention of a smeared interface. In OpenFOAM, an artificial compression term was added where the advection equation becomes:

$$
\frac{\partial \alpha}{\partial t}+\frac{\partial}{\partial x_{i}}\left(u_{i} \alpha\right)+\frac{\partial}{\partial x_{i}}\left(u_{i}^{r} \alpha(1-\alpha)\right)=0
$$

where $u^{r}$ is the compressive velocity field. By introducing this term, smear of the interface was effectively prevented. Boundness of the volume fraction was guaranteed by a special designed solver called multidimensionsal limiter for explicit solution (MULES) solver, where the bounded solution was achieved by using flux from first order upwind scheme plus a limited portion of flux from high order scheme. Detailed discretization method of Eq. (4) can be found in e.g. Berberović et al. (2009) and Márquez Damián (2013). In Gopala and van Wachem (2008) it was concluded that this VOF scheme is completely mass conservative, and as long as the Courant number is kept low, the interface can be captured sharply.

In order to obtain the equations for porous media flow, Eq. (1)-22 and Eq. (4) were ensemble averaged and volume averaged over a representative elementary volume (REV). The size of REV was much smaller than the size of the entire domain but much larger than the pore size. Therefore the volume averaged property was not dependent on the size of REV. Detailed derivation of the volume averaged equations is presented in Jensen et al. (2014) and here only the final equations are given:

$$
\begin{gathered}
\frac{\partial\left\langle\bar{u}_{i}\right\rangle}{\partial x_{i}}=0 \\
\left(1+C_{m}\right) \frac{\partial}{\partial t} \frac{\rho\left\langle\bar{u}_{i}\right\rangle}{n}+\frac{1}{n} \frac{\partial}{\partial x_{j}} \frac{\rho\left\langle\bar{u}_{i}\right\rangle\left\langle\bar{u}_{j}\right\rangle}{n}=-\frac{\partial\langle\bar{p}\rangle^{f}}{\partial x_{i}}-g_{j} x_{j} \frac{\partial \rho}{\partial x_{i}}+\frac{1}{n} \frac{\partial}{\partial x_{j}} \mu\left(\frac{\partial\left\langle\bar{u}_{i}\right\rangle}{\partial x_{j}}+\frac{\partial\left\langle\bar{u}_{j}\right\rangle}{\partial x_{i}}\right)-\frac{1}{n} \frac{\partial}{\partial x_{j}} \rho\left\langle\overline{u_{i}^{\prime} u_{j}^{\prime}}\right\rangle+S_{i} \\
\frac{\partial \alpha}{\partial t}+\frac{1}{n} \frac{\partial}{\partial x_{i}}\left(\left\langle\bar{u}_{i}\right\rangle \alpha\right)+\frac{1}{n} \frac{\partial}{\partial x_{i}}\left(\left\langle\bar{u}_{i}^{r}\right\rangle \alpha(1-\alpha)\right)=0
\end{gathered}
$$

In Eq. (6), the resistance force exerted by the porous media was represented by two terms, namely the added mass coefficient $C_{m}$, which accounts for the inertial effect due to the presence of the porous skeleton, and the drag resistance $S$. The formulation of these two forces are given below in Section 2.2 In addition, it should be mentioned that no turbulence model was applied in the present work, therefore $\rho\left\langle\overline{u_{i}^{\prime} u_{j}^{\prime}}\right\rangle=0$. The detailed reason will be illustrated in Section 4, after we introduce the expressions for porous resistance coefficients in Section 3 .

\subsection{Porous resistance force due to fishing nets}

In this section, description is given to the flow resistance force due to presence of the fishing nets. As mentioned above the resistance force was represented by two terms in Eq. 6, namely $C_{m}$ and $S$. In van Gent (1995) $C_{m}$ was expressed as:

$$
C_{m}=\gamma_{p} \frac{1-n}{n}
$$

where $\gamma_{p}$ is a nondimensional empirical coefficient, and takes value of 0.34 . This value was adopted in the present work. In the following part, we keep the main focus of the work on the drag force of the fishing net structures, and the inertia force will not be discussed until in Section 4 
The porous drag resistance was usually described by Darcy-Forchheimer equation:

$$
S_{i}=-\left(\mu D_{i j}\left\langle\bar{u}_{j}\right\rangle+\frac{1}{2} \rho C_{i j} \sqrt{\left\langle\bar{u}_{k}\right\rangle\left\langle\bar{u}_{k}\right\rangle}\left\langle\bar{u}_{j}\right\rangle\right)
$$

where $D$ and $C$ are the prescribed porous resistance coefficient matrix. In local $x^{*} y^{*} z^{*}$ coordinate where $x^{*}$ is normal to the net plane panel, $y^{*}$ and $z^{*}$ are in plane with the net panel but perpendicular to each other, they are of the following form:

$$
D_{i j}^{*}=\left[\begin{array}{ccc}
D_{1} & 0 & 0 \\
0 & D_{2} & 0 \\
0 & 0 & D_{3}
\end{array}\right] \quad C_{i j}^{*}=\left[\begin{array}{ccc}
C_{1} & 0 & 0 \\
0 & C_{2} & 0 \\
0 & 0 & C_{3}
\end{array}\right]
$$

The first term in Eq. (9), proposed in Darcy (1856), addresses a linear relation between the resistance and the volume averaged ensemble averaged velocity. The second term was added by Forchheimer (1901) to consider a nonlinear relation between them. From the porous media point of view, the relative importance of these two terms is dependent on the flow regime, which is defined based on the pore Reynolds number:

$$
R e=\frac{\langle\bar{u}\rangle d}{n \nu}
$$

In general the flow regimes are denoted as Darcy flow regime, Forchheimer flow regime, transitional flow regime and fully turbulent flow regime. For a very low Reynolds number, the linear term dominates the resistance and the quadratic term will not influence the total resistance to a very high degree. When the Reynolds number is increasing from Forchheimer regime to fully turbulent regime, the quadratic term is gaining importance. A detailed description of different flow regimes is given in Burcharth and Andersen (1995). For flow through fishing nets, in general $R e \sim \mathcal{O}\left(10^{2} \sim 10^{3}\right)$. This most probably corresponds to the fully turbulent flow regime, where the linear term is negligible and the quadratic term completely dominates over the linear term. Therefore it was reasonable to assume $D^{*}=0$. This assumption was further justified by the physical explanation: Fishing nets are composed of twines with very small diameters, typical in the order of millimeters, the quadratic drag force is the dominant force for such kind of marine structures. Inertia and other forces are secondary. (This will be illustrated in detail in Section 4). The linear term is not physical for flow through such structures, since the force should not be related to the dynamic viscosity of water. Therefore in the present numerical model, the linear drag term was completely neglected and the porous resistance force was calculated purely based on the quadratic drag term:

$$
S_{i}=-\frac{1}{2} \rho C_{i j} \sqrt{\left\langle\bar{u}_{k}\right\rangle\left\langle\bar{u}_{k}\right\rangle}\left\langle\bar{u}_{j}\right\rangle
$$

If the local $x^{*} y^{*} z^{*}$ coordinate system of the porous media is not aligned with the global $x y z$ coordinate system, the coefficient matrix needs to be transformed from local to global coordinate system. In Zhao et al. (2013a) and Patursson et al. (2010), two different methods were applied for the transformation. It was found the transformation approach in Zhao et al. (2013a) has relatively simple mathematical formulation, but it is valid only if the flow is unidirectional and the direction of the flow is aligned with $x$ axis. Patursson et al. (2010) applied a tensor transformation matrix, which is more complex but has universal applicability. The details of these two approaches are presented in Appendix A, and in the present work, the latter one was adopted.

\subsection{Forces on the net}

The instantaneous force on the net is one of the most important output from the numerical simulations. Normally for simulation of flow around ships or other offshore structures, the geometry of the structure is resolved as a boundary patch of the fluid domain, and the forces acting on them are obtained by integrating the pressure and viscous force over the boundary patch. However, when it comes to the forces acting on the porous media, the situation becomes more complex. The geometry of the structure is not resolved, therefore 
it is not possible to integrate the pressure and viscous stress on it. Patursson (2008) proposed a method based on conservation of the linear momentum in a control volume. The control volume should enclose the porous media, but the porous media does not have to fill the control volume. Furthermore the porous media could be oriented arbitrarily. So the forces acting on the fluid from the porous media is equal to the momentum loss in the control volume. The detailed derivation is illustrated in Appendix B and only the final result is presented here:

$$
Q_{i}=-\frac{\partial}{\partial t}\left(\int_{C V} \rho u_{i} \mathrm{~d} V\right)-\int_{C S} \rho u_{i} u_{j} \mathrm{n}_{j} \mathrm{~d} S-\int_{C S} p \mathrm{n}_{i} \mathrm{~d} S+\int_{C S} \tau_{i j} \mathrm{n}_{j} \mathrm{~d} S+\int_{C V} \rho g_{i} \mathrm{~d} V
$$

where $\tau_{i j}$ is the viscous stress tensor, $C V$ is the control volume and $C S$ is the control surface.

This method requires that in the numerical simulation, each term on the right-hand side of Eq. (13) must be found at every time step. In the present work another method was applied. As shown in Section 2.1 and Section 2.2 the resistance force is the force acting on the fluid from the porous media. This resistance force includes inertia and quadratic drag forces. Therefore the forces acting on the porous media should be the reaction forces of them. In the numerical model, the inertia force was neglected for the output of the forces, only the quadratic drag term was retained. This is primarily due to its difficulty to output time derivative of the velocity at run time. However in general this is acceptable since this term has minor influence in most of the cases, which will be discussed in detail in Section 4 . The final expression for the output of the instantaneous force is given as:

$$
Q_{i}=\frac{1}{2} \rho \int_{P V} C_{i j} \sqrt{\left\langle\bar{u}_{k}\right\rangle\left\langle\bar{u}_{k}\right\rangle}\left\langle\bar{u}_{j}\right\rangle \mathrm{d} V
$$

The quadratic drag term in Eq. (14) should be exactly integrated on the instantaneous wet volume of the porous media zone $P V$. In addition, the quadratic coefficient matrix $C$ may vary inside the porous media zone, therefore in Eq. (14) it should not be regarded as a constant and separated from the integrand. This usually occurs in the simulation of flow through circular gravity cages, where cylindrical coordinate system needs to be introduced as shown in Section 6.3. In local cylindrical coordinate system $C^{*}$ has the same value everywhere in the porous media zone. However after transformation into global Cartesian coordinate system, each cell has its unique value of $C$.

\section{Calculation of the quadratic drag resistance coefficients}

This section presents new formulas to calculate the quadratic coefficient matrix $C$ in Eq. (12) for flow through fishing nets. The expressions were derived based on the available rational load model. The derivation indeed reflects a transformation process from the rational load model to the porous media model, and the transformation follows the principle that the force acting on the porous media zone should be equal to the force obtained from the rational load model.

The first issue here is to choose an appropriate rational load model for the transformation. Mainly two types of load models were proposed in the existing literature. The first is Morison type load model. In this kind of model, each twine of the net is treated as an individual cylinder, and the force acting on it is calculated by Morison equation. Due to relatively high porosity for fishing nets, the interaction effects between twines are neglected. So the total forces acting on the net panel is obtained by summing up the force on each twine. This type of force model has been extensively applied in e.g. Moe et al. (2010), Xu et al. (2013a), Xu et al. (2013b) and Huang et al. (2006).

Another kind of force model is the so-called screen type force model. This model was first introduced in Løland (1993), where drag and lift force coefficients on a flat net panel were given as a function of solidity ratio and attack angle. The formulas were based on curve fitting experimental data in Rudi et al. (1988), and were suggested to be used for net panels with solidity ratio in between 0.13 and 0.317 . It was further extended in Kristiansen and Faltinsen (2012) and Kristiansen and Faltinsen (2015) to cover a wider range of solidity ratios, and the effect of Reynolds number was accounted for. 

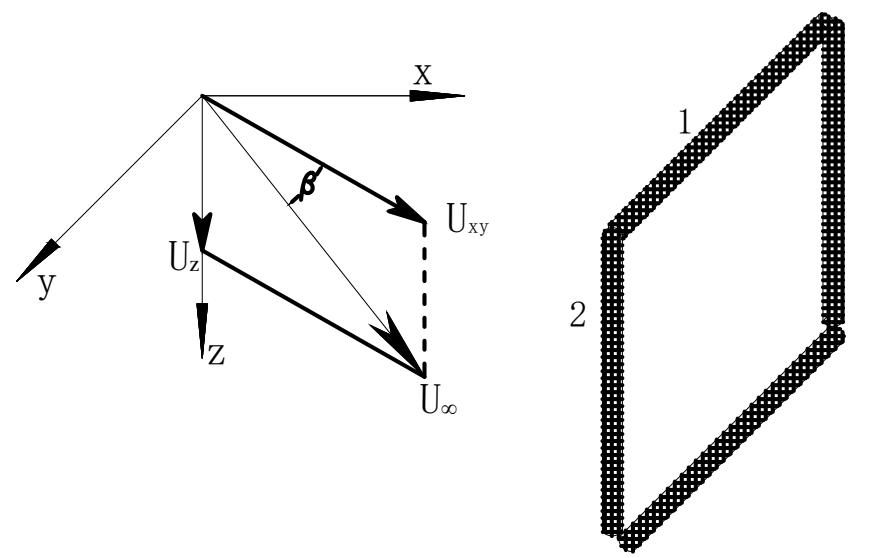

Figure 1: Left: illustration of the decomposition of the velocity vector in 3D. Right: simplification of net panel into twines without knots.

Actually both load models could be transformed to obtain the quadratic drag resistance coefficients, but with different complexities. The theory behind screen type force model is more reasonable, since the interaction effects in-between the net twines are naturally accounted for, while in Morison type load model they are neglected. However, the mathematical expressions obtained from Morison type force model have similarities with the quadratic drag force. This could greatly ease the transformation process, and lead to simple mathematical formulations. In addition, it was found that one could still obtain reasonable results by properly dropping the attack angle dependence of the derived coefficients from Morison type force model. But this will result in constant quadratic drag resistance coefficients, which is expected by the authors. This concerns the following reason: Time and space independent porous resistance coefficients could be applied in most of the open source or commercial CFD code without any modification of the code itself, as long as they support solvers for Darcy-Forchheimer type porous media flow. Therefore the derived formulas could be easily applied in most of the softwares. So it was decided to use a Morison type load model in the present paper. In order to eliminate the side effects of the assumption made in Morison type force model, i.e. there is no interaction in-between twines due to the high porosity, two new interaction coefficients $a$ and $b$ were introduced in the expressions for normal and tangential quadratic drag resistance coefficients. Physically they represent a compensation due to the above mentioned assumption. The details will be given in the following.

\subsection{Derivation}

Below in this section a generalized 3D derivation is given on the transformation of the quadratic drag resistance coefficients from Morison type load model. Assume that a fishing net panel is in steady current and only composed of twines without knots. The panel is within y-z plane and the normal direction of the net is aligned with $x$ direction, so transformation of the coefficient is not needed. The flow is ambient in $3 \mathrm{D}$ and is decomposed into components in $\mathrm{x}-\mathrm{y}$ plane and $\mathrm{z}$ axis. Fig. 1 illustrates the decomposition of the velocity vector and simplification of the net panel into small twines without knots. The purpose of such a decomposition is to simplify the 3D case into $2 \mathrm{D}$ where $U_{x y}=U_{\infty} \cos \beta$ and $U_{z}=U_{\infty} \sin \beta$. We now focus on the forces acting on net twine 1 and net twine 2 .

First we consider the velocity vector in x-y plane, namely $U_{x y}$ and its induced forces on net twines, as shown in Fig. 2. According to the cross flow principle (see e.g. Hoerner (1965)), the forces acting on twine 1 and twine 2 are different. For twine 2 the incoming flow is fully normal, and the generated force is in the 


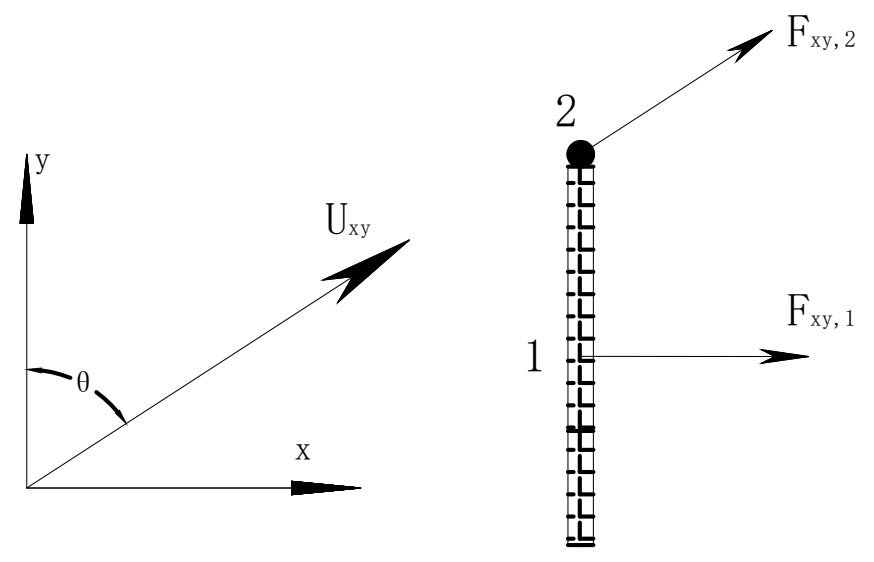

Figure 2: The projected velocity and its induced forces in $\mathrm{x}-\mathrm{y}$ plane

same direction with the flow and its magnitude is equal to:

$$
\begin{aligned}
& F_{x, 2}=\frac{1}{2} C_{d, \text { twine }} \rho A_{2} U_{x y}^{2} \sin \theta \\
& F_{y, 2}=\frac{1}{2} C_{d, \text { twine }} \rho A_{2} U_{x y}^{2} \cos \theta
\end{aligned}
$$

where $A_{2}$ is the projected area of twine 2 .

However, for twine 1 , the current velocity is decomposed into the tangential and normal components to the twine. The tangential component does not generate any forces (the friction force is small and neglected). The normal velocity generates a force aligned with $x$ direction:

$$
\begin{aligned}
& F_{x, 1}=\frac{1}{2} C_{d, t w i n e} \rho A_{1} U_{x y}^{2} \sin ^{2} \theta \\
& F_{y, 1}=0
\end{aligned}
$$

where $A_{1}$ is the projected area of twine 1.

Then we consider the forces generated by $U_{z}$. since it is fully parallel to twine 2 , no force is generated on that twine. Meanwhile it is fully perpendicular to twine 1, so generates a force aligned with $z$ direction:

$$
F_{z, 1}=\frac{1}{2} C_{d, t w i n e} \rho A_{1} U_{z}^{2}
$$

Then the total forces acting on a fishing net panel are simply superposition of the drag force for each twine:

$$
\begin{aligned}
& F_{x}=\frac{1}{2} C_{d, \text { twine }} \rho U_{x y}^{2}\left(\sin \theta \sum_{i=1}^{M} A_{2, i}+\sin ^{2} \theta \sum_{j=1}^{N} A_{1, j}\right) \\
& F_{y}=\frac{1}{2} C_{d, \text { twine }} \rho U_{x y}^{2} \cos \theta \sum_{i=1}^{M} A_{2, i} \\
& F_{z}=\frac{1}{2} C_{d, \text { twine }} \rho U_{z}^{2} \sum_{j=1}^{N} A_{1, j}
\end{aligned}
$$


where $M$ is the number of twines parallel to twine 2 , or the so-called out-of-plane twin. $N$ is the number of twines parallel to twine 1 , which is the in-plane twine.

On the other hand, according to Section 2.3 the forces acting on the porous media $Q$ were obtained by applying Eq. (14):

$$
\begin{aligned}
Q_{x} & =\frac{1}{2} \rho C_{1} V U_{\infty} U_{x y} \sin \theta \\
Q_{y} & =\frac{1}{2} \rho C_{2} V U_{\infty} U_{x y} \cos \theta \\
Q_{z} & =\frac{1}{2} \rho C_{3} V U_{\infty} U_{z}
\end{aligned}
$$

Here it was assumed that the unknown volume averaged velocity $\langle\bar{u}\rangle$ is equal to the undisturbed velocity. This is a reasonable assumption since fishing net is kind of porous structure with very high porosity, and the velocity reduction is not significant, as shown in Bi et al. (2013a). Therefore the volume averaged ensemble averaged velocity $\langle\bar{u}\rangle$ inside the porous media should be very close to the undisturbed velocity.

Since the fishing nets were approximated by a sheet of porous media, the forces acting on the porous media should be equal to the superposition of forces from each twine, i.e. $F=Q$. By substituting the expression of $F$ and $Q$ into the equilibrium relation, the following relations were obtained for $C_{1}, C_{2}$ and $C_{3}$ :

$$
\begin{aligned}
C_{1} & =\frac{1}{V} C_{d, \text { twine }} \cos \beta\left(\sum_{i=1}^{M} A_{2, i}+\sin \theta \sum_{j=1}^{N} A_{1, j}\right) \\
C_{2} & =\frac{1}{V} C_{d, \text { twine }} \cos \beta \sum_{i=1}^{M} A_{2, i} \\
C_{3} & =\frac{1}{V} C_{d, \text { twine }} \sin \beta \sum_{j=1}^{N} A_{1, j}
\end{aligned}
$$

As mentioned at the beginning of this section, two coefficients $a$ and $b$ were introduced to account for the interaction effects in-between twines in tangential and normal direction, respectively. In addition, the derived expressions for $C$ as shown above have attack angle dependence, indicating time and space varying quadratic drag resistance coefficients in unsteady flow. However, in traditional porous media theory, $C$ was applied as a constant for all the attack angles. Angle dependence of porous resistance was merely handled by transformation from local to global coordinate system, while porous resistance coefficients were kept the same. Recalling that in the literature survey in Section 1, almost all the paper applied constant porous resistance coefficients, but still obtained good agreement between numerical and experimental results. This indicates that angle dependence of the porous resistance coefficients might have minor effects on the final results. Therefore in the present work, it was directly dropped, and constant drag resistance coefficients were applied throughout the paper. Actually this further indicated that an averaged value of $C$ against different attack angles was employed, and this contribution was implicitly included in the parameters of $a$ and $b$. This leads to the final expressions for the coefficients:

$$
\begin{aligned}
C_{1} & =\frac{1}{V} a C_{d, \text { twine }}\left(S_{1}+S_{2}\right) \\
C_{2} & =\frac{1}{V} b C_{d, \text { twine }} S_{2} \\
C_{3} & =\frac{1}{V} b C_{d, \text { twine }} S_{1}
\end{aligned}
$$

where $S_{1}$ is the total projected area for in-plane twines where $S_{1}=\sum_{j=1}^{N} A_{1, j}, S_{2}$ is the total projected area for out-of-plane twines where $S_{2}=\sum_{i=1}^{M} A_{2, i}$. Given Eq. $30-32$, the forces acting on the porous media 
were expressed as:

$$
\begin{aligned}
Q_{x} & =\frac{1}{2} \rho a C_{d, \text { twine }} U_{\infty}^{2}\left(S_{1}+S_{2}\right) \cos \beta \sin \theta \\
Q_{y} & =\frac{1}{2} \rho b C_{d, \text { twine }} U_{\infty}^{2} S_{2} \cos \beta \cos \theta \\
Q_{z} & =\frac{1}{2} \rho b C_{d, \text { twine }} U_{\infty}^{2} S_{1} \sin \beta
\end{aligned}
$$

A few additional comments are given here on the interaction coefficients $a$ and $b$ : (1) When considering flow through nets, mainly two interaction effects should be accounted for, namely the shading effect of the downstream twines from the upstream twines for large inflow angles, and the local speed-up of the flow in-between the twines. This has been explained in detail in Kristiansen and Faltinsen (2012). Both of them are affected by the solidity ratio, therefore $a$ and $b$ were believed to be functions of solidity ratio. (2) The parameter $a$, which is in the expression for the normal quadratic drag resistance coefficient $C_{1}$, is strongly influenced by the local speed-up interaction effect. Therefore it is expected that when $S_{n} \rightarrow 0$, where the distance between the twines is infinitely large, the flow should not speed up and $a \rightarrow 1$. (3) Both tangential porous resistance coefficients $C_{2}$ and $C_{3}$ share the same interaction coefficient $b$. This is reasonable since for most of the fishing nets, the material properties in $y$ and $z$ direction, (i.e. within the net panel plane) are the same. Therefore the interaction effects in both directions should be the same. Indeed for most of the fishing nets, especially the nets used in aquaculture industry, $C_{2} \approx C_{3}$ since they usually have symmetric mesh patterns. In previous works, they were usually represented by a single tangential resistance coefficient called $C_{t}$. (4) For parameter $b$, which accounts for the flow interaction effects for tangential porous resistance coefficient, both interaction effects may play important roles. Shading effect will result in a reduction of the force on the downstream twine due to decrease of the incoming velocity. But the local speed-up effect will lead to an increase of the force on the twine. (5) By comparison of the results between CFD simulations and experiments, it was found that for nets with small solidity ratios, $b$ was almost kept as a constant. Meanwhile, it started to increase for nets with intermediate to high solidity ratios. This indicated that both effects are equally important for nets with small solidity ratios but the local speed-up effect is gaining importance when solidity ratio increases.

\subsection{Calibration of the parameters}

In this section, the unknown interaction coefficients $a$ and $b$ were calibrated for nets with different solidity ratios. The reference values for three specific nets were determined first based on the selected experimental data in Rudi et al. (1988). The principle for determination of $a$ and $b$ is that the error between the drag and lift forces obtained from Eq. (33- 34) and from laboratory tests should be minimized. (The out-of-plane lift force $Q_{z}$ was not considered here due to $2 \mathrm{D}$ flow in Rudi et al. (1988), i.e. $\beta=0^{\circ}$.) The error function $E$ was defined based on the least square normalized error:

$$
E=\sum_{p=1}^{P}\left(\frac{F_{x, p}^{M}-Q_{x, p}}{F_{x, p}^{M}}\right)^{2}+\sum_{k=1}^{K}\left(\frac{F_{y, k}^{M}-Q_{y, k}}{F_{y, k}^{M}}\right)^{2}
$$

where $F^{M}$ is the measured force with different incoming velocities and attack angle for one specific net. $P$ and $K$ are the number of data used in calibration for forces in $x$ and $y$ direction, respectively. Substituting Eq. (33) and Eq. (34) into Eq. (36) reads:

$$
E=\sum_{p=1}^{P}\left(1-\frac{1}{2 F_{x, p}^{M}} \rho a C_{d, t w i n e}^{p} U_{\infty, p}^{2} \sin \theta_{p}\left(S_{1}+S_{2}\right)\right)^{2}+\sum_{k=1}^{K}\left(1-\frac{1}{2 F_{y, k}^{M}} \rho b C_{d, t w i n e}^{k} U_{\infty, k}^{2} \cos \theta_{k} S_{2}\right)^{2}
$$

As mentioned above, the incoming velocity $U_{\infty}$, attack angle $\theta$ and drag coefficient $C_{d, t w i n e}$ are varying with index of the experimental data $p$ and $k$. By taking derivative of $E$ with respect to $a$, and equaling it 
to zero, $a$ was expressed as:

$$
a=\frac{A_{n e t} \sum_{p=1}^{P}\left(\frac{C_{d, t w i n e}^{p} \sin \theta_{p}}{C_{d, n e t}^{p}}\right)}{\left(S_{1}+S_{2}\right) \sum_{p=1}^{P}\left(\frac{\sin ^{2} \theta_{p}\left(C_{d, t w i n e}^{p}\right)^{2}}{\left(C_{d, n e t}^{p}\right)^{2}}\right)}
$$

By the same manipulation $b$ was obtained:

$$
b=\frac{A_{n e t} \sum_{k=1}^{K}\left(\frac{C_{d, t w i n e}^{k} \cos \theta_{k}}{C_{l, n e t}^{k}}\right)}{S_{2} \sum_{k=1}^{K}\left(\frac{\cos ^{2} \theta_{k}\left(C_{d, t w i n e}^{k}\right)^{2}}{\left(C_{l, n e t}^{k}\right)^{2}}\right)}
$$

where the drag and lift force coefficients of the net panel were defined as

$$
\begin{aligned}
C_{d, n e t} & =\frac{F_{d, n e t}}{1 / 2 \rho A_{n e t} U_{\infty}^{2}} \\
C_{l, n e t} & =\frac{F_{l, n e t}}{1 / 2 \rho A_{n e t} U_{\infty}^{2}}
\end{aligned}
$$

In Rudi et al. (1988) series of experiments were conducted under a variety of velocities and attack angles for nets with different solidity ratios. The experimental data for three net cases were selected to calibrate the formula and their geometrical characteristics are listed in Table 1. The reason to select these three nets is that extensive experiments have been done in Rudi et al. (1988) for these three net panels, and plenty of data are available. In addition the solidity ratio of these three nets are distributed from low to high, providing the reference value of $a$ and $b$ in a wide range of net panels. The calculated reference values of the interaction coefficients are also presented in Table 1

Table 1: The geometric parameters of the three nets in Rudi et al. (1988) and the calibrated reference values of the interaction coefficients

\begin{tabular}{cccc}
\hline Net case no. & 1 & 2 & 3 \\
\hline Solidity ratio & 0.13 & 0.243 & 0.317 \\
Twine diameter $(\mathrm{mm})$ & 1.83 & 1.83 & 1.83 \\
Bar length $(\mathrm{mm})$ & 29.0 & 15.5 & 12 \\
$\mathrm{a}$ & 1.31 & 1.45 & 1.85 \\
$\mathrm{~b}$ & 0.92 & 0.85 & 1.50 \\
\hline
\end{tabular}

Linear interpolation was applied to obtain the interaction coefficients for nets with other solidity ratios. This considers the boundness property of linear interpolation, i.e. for a net panel with solidity ratio inbetween the solidity ratios for the reference nets, the interaction coefficients $a$ and $b$ should also remain in-between the values of the coefficients for the reference nets. Polynomial fit may introduce unexpected extroplative behavior. For $0<S_{n}<0.13$, asymptotic value of $a$ was used while $b$ was kept constant. This has been explained in Section 3.1. The final expression of $a$ and $b$ is given in Eq. (42) and Eq. 43.

$$
\begin{gathered}
a=\left\{\begin{array}{lc}
2.3484 S_{n}+1 & 0<S_{n} \leq 0.13 \\
1.3128 S_{n}+1.1346 & 0.13<S_{n} \leq 0.243 \\
5.3094 S_{n}+0.1634 & 0.243<S_{n} \leq 0.317
\end{array}\right. \\
b=\left\{\begin{array}{lc}
0.9241 & 0<S_{n} \leq 0.13 \\
-0.6310 S_{n}+1.0061 & 0.13<S_{n} \leq 0.243 \\
8.7581 S_{n}-1.2754 & 0.243<S_{n} \leq 0.317
\end{array}\right.
\end{gathered}
$$




\section{Discussions on the overall numerical model}

So far the description of the numerical model for flow through fishing nets is completed, and below discussions will be given on several topics related to our numerical model. This includes the discussion of VARANS equations and its application in the present model, the inertia effect for flow through fishing nets, the turbulent effects and the reason why no turbulence model was applied in the present model.

\subsection{Comments on solid-fluid interaction effects in VARANS equations}

In the present model, VARANS equations derived from Jensen et al. (2014) were employed as the governing equations. Meanwhile we notice that in previous works, e.g. Patursson et al. (2010), a different mathematical formulation of porous media flow was used:

$$
\begin{gathered}
\frac{\partial u_{i}}{\partial x_{i}}=0 \\
\frac{\partial \rho u_{i}}{\partial t}+\frac{\partial \rho u_{i} u_{j}}{\partial x_{j}}=-\frac{\partial P}{\partial x_{i}}+\frac{\partial}{\partial x_{j}} \mu\left(\frac{\partial u_{i}}{\partial x_{j}}+\frac{\partial u_{j}}{\partial x_{i}}\right)+S_{i}
\end{gathered}
$$

The main discrepancies between Eq. (44- 45) and Eq. (5)-6) lie in the inertia term, and the porosity was not included in Eq. (45). The added mass coefficient will be discussed in Section 4.2 and here it is focused on the influences of including porosity in the governing equations. Actually Eq. 45 implicitly indicated that the porosity of the fishing nets was equal to one. Therefore it was assumed that the volume of the net was neglected, and water was filled in the domain everywhere. The only effect of the net was the resistance force. Essentially instead of volume averaging Eq. (1-2), only one extra resistance term was added. This could be considered as a reasonable simplification for clean net, which has a very high porosity value, normally between 0.7 and 0.9 . However for nets with biofouling, as shown in Lader et al. (2015), the porosity could be reduced to a very low value. Then this simplification is questionable. However, in the present model, the effect of porosity i.e. net-fluid interaction was neither fully considered, as shown in the derivation of the resistance term $S$. In Section 3.1, it was seen that the fundamental assumption behind Morison type load model is that due to relatively high porosity, the interaction effects in-between the twines are neglected. This indicated that the load on each twine was calculated individually first, assuming in the infinite fluid domain without the presence of its neighboring twines. Then this was compensated by introducing the interaction coefficients $a$ and $b$, which are determined based on the experimental data.

\subsection{Inertia force for flow through fishing nets}

The inertia effect due to presence of the porous skeleton was not accounted for in Eq. (45). But in Eq. (6), this was considered in a generic way, i.e. only as a function of porosity and irrespective the details of the porous skeleton. Previous works show that this term has minor effects in most of the cases, e.g. in del Jesus (2011) and Jacobsen et al. (2015). Actually the inertia force on a fishing net panel should be a small part of the total force, since the ratio between magnitude of inertia force and drag force for a twine of unit length in periodic unsteady flow was expressed as (see Eq. (4.31) in Sumer and Fredsøe (2006)):

$$
\frac{F_{I, \text { twine }}}{F_{d, \text { twine }}}=\frac{C_{I, t \text { twine }} \pi d^{2} \omega U_{m}}{2 C_{d, \text { twine }} d U_{m}^{2}}=\frac{\pi^{2}}{K C} \frac{C_{I, \text { twine }}}{C_{d, \text { twine }}}
$$

For net twines whose diameter is normally a few millimeters, $\mathrm{KC}$ number is in the order of hundred while $C_{I} / C_{D} \approx 2$. So the ratio was expected to be small. Although in Balash et al. (2009) and Lader et al. (2007a), it was reported that inertia force may be important for nets with high solidity ratios, in the present work we focus on the drag force, and the inertia term was just inherited from Jensen et al. (2014) without further investigations. 


\subsection{Turbulence effects for flow through fishing nets}

As shown in Section 2.2, for flow through net structures, it is most probably in the fully turbulent regime. Therefore turbulence is generated in the wake, which is also shown in Bouhoubeiny et al. (2011), Pichot et al. (2009), Kim (2012) and Lader et al. (2007b). However in the numerical model, the geometry of the net was not resolved, so the boundary layers of the twines and knots were not able to be modeled. Instead, the turbulence level in the porous media was represented by $-\rho\left\langle\overline{u_{i}^{\prime} u_{j}^{\prime}}\right\rangle$ in Eq. $[6$. Ideally this term should be taken into account by a turbulence model. By applying Boussinesq assumption, this term can be approximated as:

$$
-\rho\left\langle\overline{u_{i}^{\prime} u_{j}^{\prime}}\right\rangle=\left\langle\mu_{t}\right\rangle\left(\frac{\partial\left\langle\bar{u}_{i}\right\rangle}{\partial x_{j}}+\frac{\partial\left\langle\bar{u}_{j}\right\rangle}{\partial x_{i}}\right)-\frac{2}{3} \rho\langle k\rangle \delta_{i j}
$$

where $\delta_{i j}$ yields Einstein summation and $\delta_{i i}=\delta_{11}+\delta_{22}+\delta_{33}=3$.

This requires that the equations involved in the applied turbulence model should also be volumed averaged. Nakayama and Kuwahara (1999) presented how the transport equations for turbulence kinetic energy and dissipation rate were volume averaged for a $k-\epsilon$ turbulence model. However by following the averaging procedure, some extra unknown terms were introduced representing generation and dissipation of the turbulence kinetic energy. They were determined by numerical experiments to close the system.

For flow through fishing nets, due to its relatively high porosity, in general most of the turbulence can be freely convected through the nets. Only large scale turbulence whose length scale is larger than the mesh bar length will be damped, while small vortex will be generated in the wake of the twines.

However, it was decided not to apply any turbulence model in the present work, i.e. $\left\langle\mu_{t}\right\rangle=0$ and $\langle k\rangle=0$. This concerns that the actual level of turbulence kinetic energy was of minor interests. Meanwhile the flow resistance term $S$, which was described by Darcy-Forchheimer equation, was introduced to the Navier-Stokes equations as a closure model for handling the porous drag force which cannot be resolved directly in the model. This also corresponds to the concept of a closure model for turbulence modeling. In Section 3 it was shown that the quadratic drag resistance coefficients were written as a function of physical parameters of the fishing nets, and also the so-called interaction coefficient $a$ and $b$. They were actually calibrated based on the measurements, which already included all the dissipative effects including turbulence. Application of a turbulence model may introduce dual turbulence dissipation. This is considered to be a valid approximation in many engineering applications and it was also applied in Jensen et al. (2014) and Jacobsen et al. (2015). However one should notice that by doing so, the flow in the wake was not described in a correct way by the numerical model, where the turbulence generated behind the fishing nets was not resolved. When studying the circular fish cages, this may affect the analysis in the rear part of the cage.

\section{Preliminary tests on convergence analysis}

Series of preliminary tests have been run for convergence analysis. The purpose is to find an appropriate mesh resolution for CFD simulations. In addition, for net cages in steady current, analysis was also performed to examine the convergence property in time domain. Some of the conclusions made in Patursson et al. (2010) were also applied in the present model.

Hexahedral mesh was adopted in the simulation where the mesh was refined in the near-net region. The overall aspect ratio was kept between one to two, and the mesh grading was smoothened from far field to the near field. Convergence property of the mesh was studied for cases in Patursson et al. (2010) with $\theta=90^{\circ}$ and different incoming velocities $U_{\infty}$. The detailed setup of the numerical model will be given in Section 6.1, and the results for the convergence study are shown in Fig. 3. Here the resolution of the mesh was represented by the number of the layers $N$ used in the porous zone. The exact numerical solution was not known, and the absolute error vector was expressed as the difference between the solutions from the current mesh resolution and the finest mesh resolution for different incoming velocities. The relative error was computed as the second norm of the ratio between absolute error and solutions from the finest mesh resolution, and it is also shown in Fig. 3 It was concluded that the mesh resolution is important for the numerical solution, and the numerical solution is monotonic against mesh resolution, indicating that it is converging towards an exact numerical solution. However, the difference between the numerical results from 

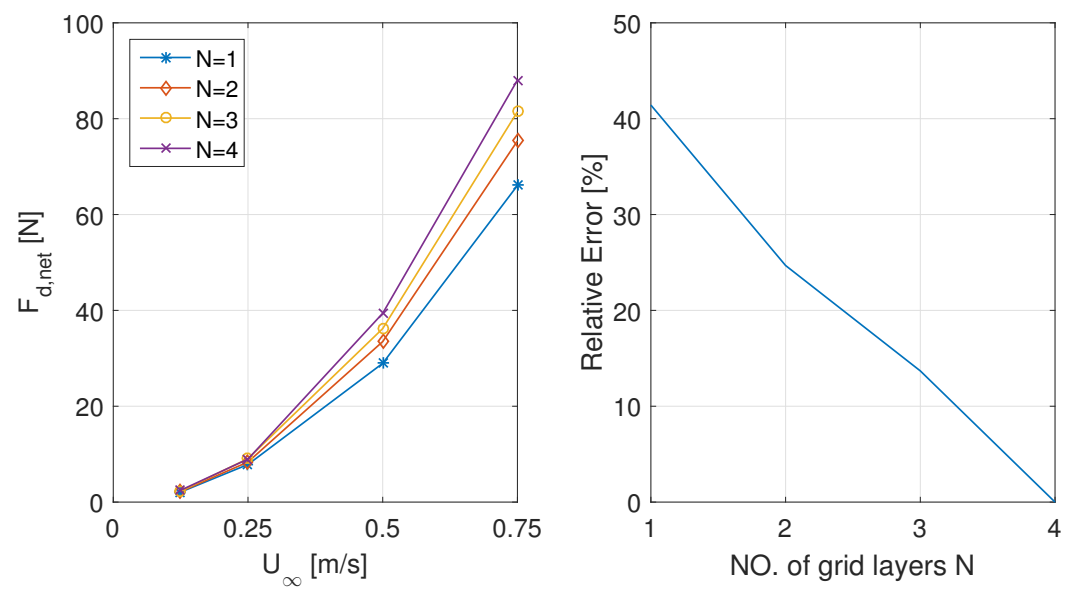

Figure 3: Convergence study for cases in Patursson (2007) with $\theta=90^{\circ}$ and different incoming velocities $U_{\infty}$. The left shows the absolute drag force as a function of incoming velocities for different solidity ratios. The right gives the relative error as a function of mesh resolution.

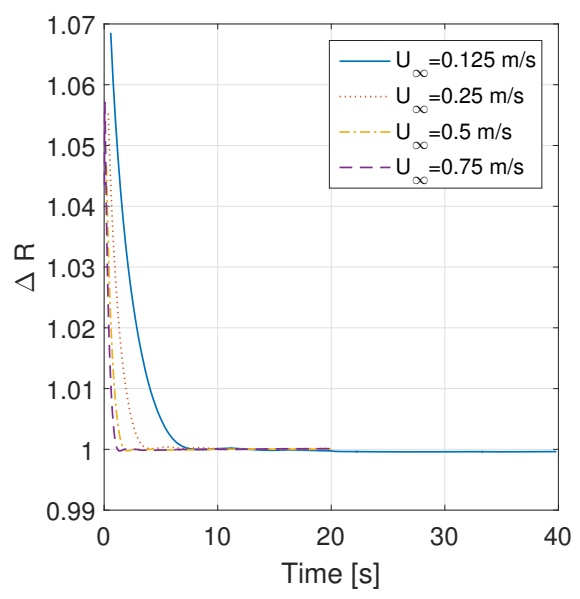

Figure 4: An example of convergence rate for numerical simulation of cases with $\theta=90^{\circ}$ in Patursson et al. (2010).

two finest mesh resolutions is around $12 \%$. Therefore we could not conclude that the numerical solution was converged. Compromises were made between the computational cost and accuracy of the numerical solution, and it was decided to use a mesh resolution of $N=3$ for all the validation cases.

The convergence property in time domain was investigated for fishing nets in steady current. It was found that in general the forces got converged in a rapid speed. An example of convergence in time domain is presented in Fig. 4. where numerical simulations were also carried out for cases in Patursson et al. (2010). In Fig. 4 the ratio $\Delta R$ between the instantaneous drag forces and the converged drag forces is plotted as a function of time for the flow with $\theta=90^{\circ}$ and four different incoming velocities. For all the four cases, the forces converged at $t=10 \mathrm{~s}$, and the convergence rate is proportional to the inverse of the incoming velocities. In Section 6, care was taken to make sure that the probed data were fully converged for the validation cases for nets in steady current.

In Patursson et al. (2010) some conclusions have been drawn on general setup of the numerical model, and the most important conclusion is that, as long as guidelines based on the physical consideration were adhered to, the model was remarkably insensitive to many of the specific choices. The thickness of the porous media representing the net was $50 \mathrm{~mm}$ in Patursson et al. (2010), and it was found that this parameter does not affect the simulation significantly. Therefore the same thickness was used throughout the paper. Effects 
Table 2: The selected drag force coefficients for the net twines and the calculated quadratic drag resistance coefficients for the net panel used in Patursson et al. (2010) under different incoming velocities

\begin{tabular}{ccccc}
\hline$U[\mathrm{~m} / \mathrm{s}]$ & 0.125 & 0.25 & 0.5 & 0.75 \\
\hline$R e$ & 350 & 700 & 1400 & 2100 \\
$C_{d, \text { twine }}$ & 1.35 & 1.15 & 1.00 & 1.00 \\
$C_{1}$ & 7.18 & 6.12 & 5.32 & 5.32 \\
$C_{2}$ & 2.32 & 1.98 & 1.72 & 1.72 \\
$C_{3}$ & 2.32 & 1.98 & 1.72 & 1.72 \\
\hline
\end{tabular}

of near wall treatment was also investigated in Patursson (2008). It was concluded that no significant difference was found by using different near wall treatment. In the present work, both slip and no-slip boundary condition were applied in different validation cases.

\section{Validation of the numerical model}

In this section, the overall numerical model is validated thoroughly against laboratory tests. The validation cases include both plane net panels and also circular net cages. The cases were carried out with the focus on current and wave loads on fishing nets under a variaty of conditions. The selected net panels cover a wide range of solidity ratios in order to demonstrate the universal applicability of the numerical model.

\subsection{Current interaction with plane net panel: validation case 1}

The first validation case is based on the experimental data presented in Patursson (2007) for a plane net panel in current flow under various attack angles and incoming velocities. The experiments were conducted in the towing tank in University of New Hampshire (UNH) in United States. The towing tank is $37 \mathrm{~m}$ long, $3.66 \mathrm{~m}$ wide and $2.44 \mathrm{~m}$ deep. The net panel was positioned in the center of the cross section and well below the water surface. A rigid frame was used to hold the net so the deformation was negligible. The drag and lift forces on the net panel, and the velocity at $2.5 \mathrm{~m}$ behind the net panel were measured. The velocity reduction factor $U_{r}$ was calculated as

$$
U_{r}=\frac{U_{\infty}-U_{2.5}}{U_{\infty}}
$$

where $U_{2.5}$ is the velocity at $2.5 \mathrm{~m}$ behind the net panel.

The net used in the experiments was a $1 \mathrm{~m}$ by $1 \mathrm{~m}$ knotless nylon net with $d=2.8 \mathrm{~mm}$ and $\lambda=29 \mathrm{~mm}$. This gave a solidity ratio of 0.184 . The measurements were performed at the attack angle of $0^{\circ}, 15^{\circ}, 30^{\circ}$, $45^{\circ}, 60^{\circ}$ and $90^{\circ}$ and under the towing speed of $12.5,25,50,75 \mathrm{~cm} / \mathrm{s}$.

A three dimensional numerical model was set up based on the experiments. The sketch of domain and the measurement point are presented in Fig. 5. According to the mesh bar length and diameter of the twines of the net panel, the porous resistance coefficients were calculated based on Eq. (30-32), with the parameters $a$ and $b$ obtained from Eq. (42-43). The calculated coefficients are listed in Table 2 with the drag coefficients of the twines chosen from Schlichting and Gersten (2003).

The velocity contours at a variety of attack angles from the CFD simulations are plotted in Fig. 6 for $U_{\infty}=0.5 \mathrm{~m} / \mathrm{s}$. In general it has the same characteristics with the flow field shown in Patursson et al. (2010). In cross flow direction, the width of the wake is approximately the same with the net panel. Meanwhile along the flow direction, the reduction of the flow speed starts a short distance in front of the plane net panel, and finally reaches the steady state behind the net panel. But we also notice some differences on the near flow field behind the net panel. In Patursson et al. (2010) a continuous process of reduction was observed, and the distance from the net panel to the start point of the steady flow speed level is approximately one to two times the width of the net panel. But in the present simulations, a very rapid process of the reduction was seen. It might be due to the difference in model setups. In Patursson et al. (2010), the frame was also modeled as small diameter cylinders. But in the present model, it was neglected. 

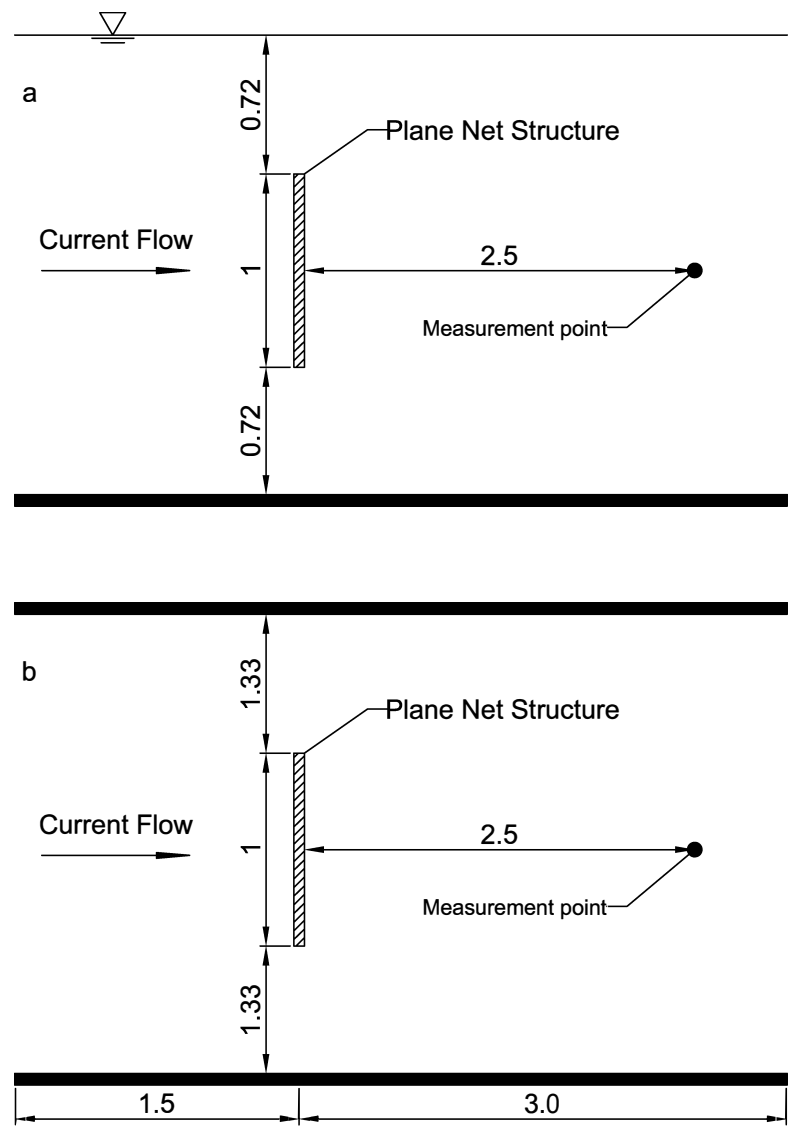

Figure 5: Sketch of the domain for reproduction of the experiments in Patursson (2007). (a) side view. (b) top view. (unit: $\mathrm{m})$

The drag and lift force coefficients, and the velocity reduction factor calculated from the experimental data in Patursson (2007) and the present numerical model in OpenFOAM are shown in Fig. 7 . In addition, the results from Patursson et al. (2010) based on the fitting procedure with different error functions, namely the least square absolute normalized error function (LANE), least square absolute error function (LAE), and least square normalized error function (LSNE) are also presented in Fig. 7. The associated relative error is presented in Fig. 8 .

Regarding the drag force coefficients, the present model gave similar predictions with fitting methods. For cases with $U_{\infty}=0.25 \mathrm{~m} / \mathrm{s}, U_{\infty}=0.5 \mathrm{~m} / \mathrm{s}$ and $U_{\infty}=0.75 \mathrm{~m} / \mathrm{s}$, the drag force coefficients at $\theta=90^{\circ}$ were slightly underestimated by $10 \%$ by all the methods. The relatively large errors at $\theta=0$ were induced by the small absolute values of the drag force coefficients. However, there appears discrepancy between the present model and fitting methods for prediction of lift force coefficients at $U_{\infty}=0.125 \mathrm{~m} / \mathrm{s}$. Better predictions were given by fitting methods in general, where the present model overestimated the lift force coefficients by $20 \%-30 \%$, except at $\theta=60^{\circ}$. For cases with the remaining incoming velocities, they have similar performance. The nonzero lift force at $\theta=90^{\circ}$ from experiments may be due to the anti-symmetries in the net panel as explained in Patursson et al. (2010), and this results in significant relative errors as shown in Fig. 8. Regarding wake velocity, significant discrepancies were observed between CFD simulations by all the methods and experimental data at small attack angles. In Patursson et al. (2010) the reasons were explained: In the experiment the high velocity in the wake was due to the effect from the frame, while in CFD model the effect from the frame was small (in the numerical model of Patursson et al. (2010)) or nonexistent (in the present model). However, it appears that the present model gave much more reasonable 

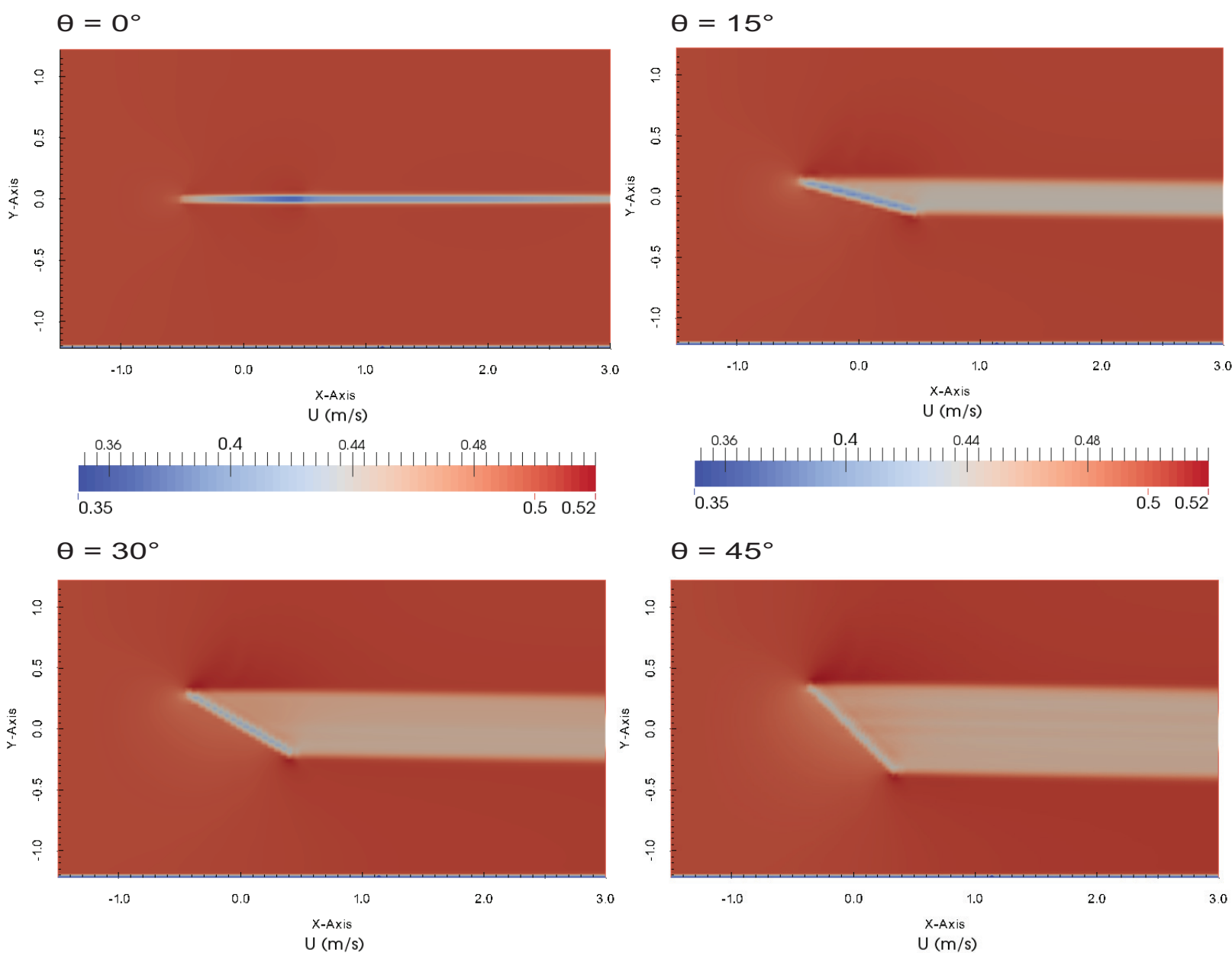

$$
\theta=45^{\circ}
$$
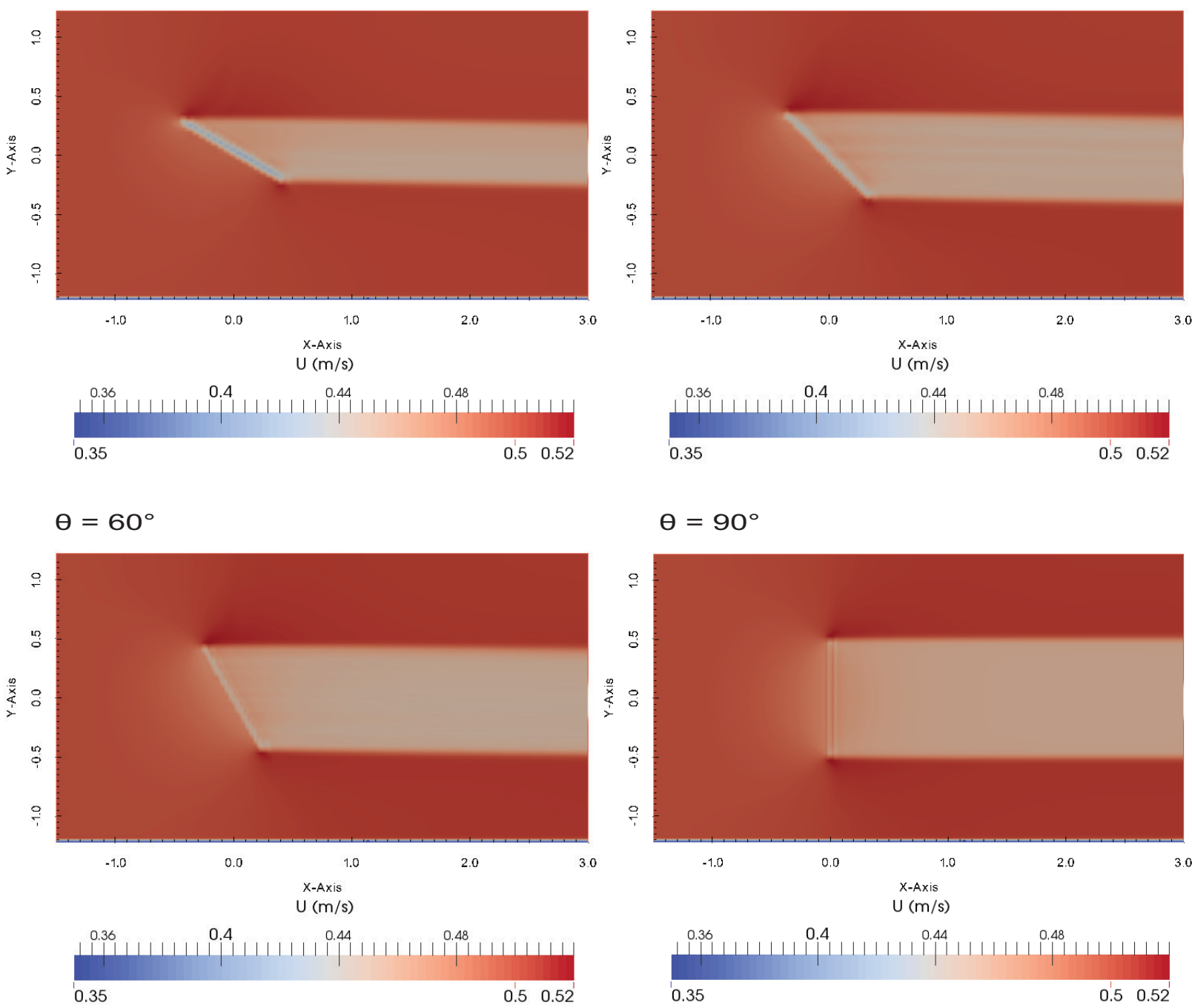

Figure 6: The velocity contours from the present numerical model on a vertical plane cut through the center of the net panel at different angles of attack for incoming velocity of $0.5 \mathrm{~m} / \mathrm{s}$. 
Table 3: The physical parameters of the net panels in Zhan et al. (2006), and calculation of the corresponding porous resistance coefficients

\begin{tabular}{|c|c|c|c|c|}
\hline Net case & & 1 & 2 & 3 \\
\hline$\lambda(\mathrm{m})$ & & 0.0125 & 0.0065 & 0.0130 \\
\hline$d(\mathrm{~m})$ & & 0.0008 & 0.0007 & 0.0015 \\
\hline$S_{n}$ & & 0.128 & 0.215 & 0.223 \\
\hline$S_{1}\left(\mathrm{~m}^{3}\right)$ & & 0.0588 & 0.0988 & 0.1028 \\
\hline$S_{2}\left(\mathrm{~m}^{3}\right)$ & & 0.0593 & 0.0992 & 0.1037 \\
\hline$V\left(\mathrm{~m}^{3}\right)$ & & 0.0455 & 0.0455 & 0.0455 \\
\hline$a$ & & 1.30 & 1.42 & 1.43 \\
\hline$b$ & & 0.93 & 0.87 & 0.87 \\
\hline \multirow{5}{*}{$U_{\infty}=0.25 \mathrm{~m} / \mathrm{s}$} & $R e$ & 200 & 175 & 362 \\
\hline & $C_{d, t w i n e}$ & 1.55 & 1.60 & 1.35 \\
\hline & $C_{1}$ & 5.23 & 9.86 & 8.74 \\
\hline & $C_{2}$ & 1.87 & 3.04 & 2.66 \\
\hline & $C_{3}$ & 1.85 & 3.02 & 2.64 \\
\hline \multirow{5}{*}{$U_{\infty}=0.5 \mathrm{~m} / \mathrm{s}$} & $R e$ & 400 & 350 & 725 \\
\hline & $C_{d, \text { twine }}$ & 1.30 & 1.35 & 1.10 \\
\hline & $C_{1}$ & 4.39 & 8.32 & 7.13 \\
\hline & $C_{2}$ & 1.57 & 2.56 & 2.17 \\
\hline & $C_{3}$ & 1.55 & 2.55 & 2.15 \\
\hline \multirow{5}{*}{$U_{\infty}=0.75 \mathrm{~m} / \mathrm{s}$} & $R e$ & 600 & 525 & 1080 \\
\hline & $C_{d, t w i n e}$ & 1.18 & 1.20 & 1.00 \\
\hline & $C_{1}$ & 3.98 & 7.40 & 6.48 \\
\hline & $C_{2}$ & 1.42 & 2.28 & 1.97 \\
\hline & $C_{3}$ & 1.41 & 2.27 & 1.96 \\
\hline \multirow{5}{*}{$U_{\infty}=1 \mathrm{~m} / \mathrm{s}$} & $R e$ & 800 & 700 & 1450 \\
\hline & $C_{d, t w i n e}$ & 1.10 & 1.15 & 1.00 \\
\hline & $C_{1}$ & 3.71 & 7.09 & 6.48 \\
\hline & $C_{2}$ & 1.32 & 2.18 & 1.97 \\
\hline & $C_{3}$ & 1.31 & 2.17 & 1.96 \\
\hline
\end{tabular}

results for the wake velocity at $U_{\infty}=0.125 \mathrm{~m} / \mathrm{s}$ and $U_{\infty}=0.25 \mathrm{~m} / \mathrm{s}$ at $\theta=0^{\circ}$.

\subsection{Current interaction with plane net panel: validation case 2}

Further validation cases were carried out for plane net panel in steady current. In order to cover wider range of different net panels, we selected the experiments performed in Zhan et al. (2006) to validate our numerical model. The experiments were conducted in the towing tank at the Department of Applied Mechanics, Zhongshan University in China. The towing tank has a dimension of $204 \mathrm{~m} \times 6 \mathrm{~m} \times 3 \mathrm{~m}$. The net panel was $1.3 \mathrm{~m}$ long and $0.7 \mathrm{~m}$ high, and tightly fixed in the frame where the deformation was negligible. The drag force for the net panel was measured under attack angles of $30^{\circ}, 60^{\circ}$ and $90^{\circ}$. Four towing speeds were applied in the experiments, namely $0.25 \mathrm{~m} / \mathrm{s}, 0.5 \mathrm{~m} / \mathrm{s}, 0.75 \mathrm{~m} / \mathrm{s}$ and $1 \mathrm{~m} / \mathrm{s}$.

Totally three kinds of net panels with different solidity ratios were studied in the experiments. The mesh for all the three nets were square diamond pattern. The physical parameters of the net panels are listed in Table 3, with the associated parameters for calculation of the quadratic drag resistance coefficients.

The sketch of the domain for numerical model is presented in Fig. 9. The depth of the domain was reduced in the numerical model. Moreover, slip condition was applied for the bottom wall. The main reason behind this setup is that the bottom should have negligible effects on flow through the net panels. This is beneficial in the perspective of computational time. 
a

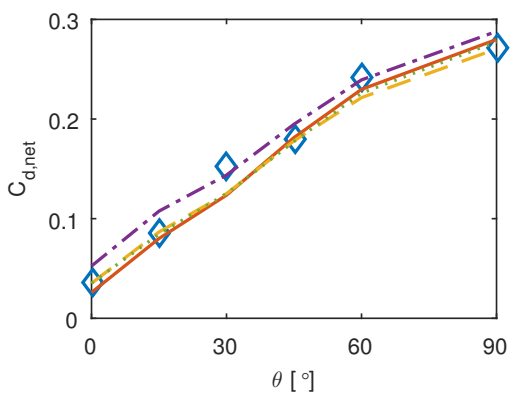

b

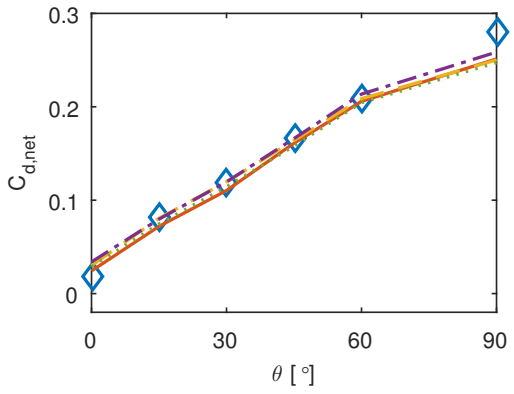

C

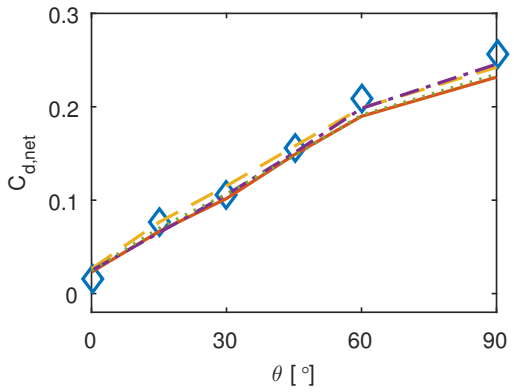

d

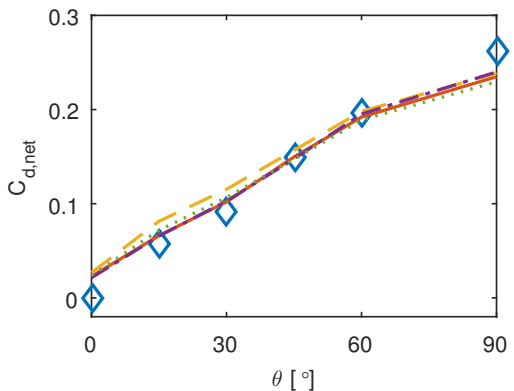

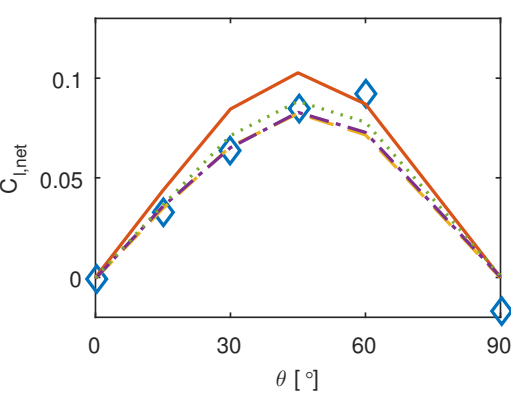
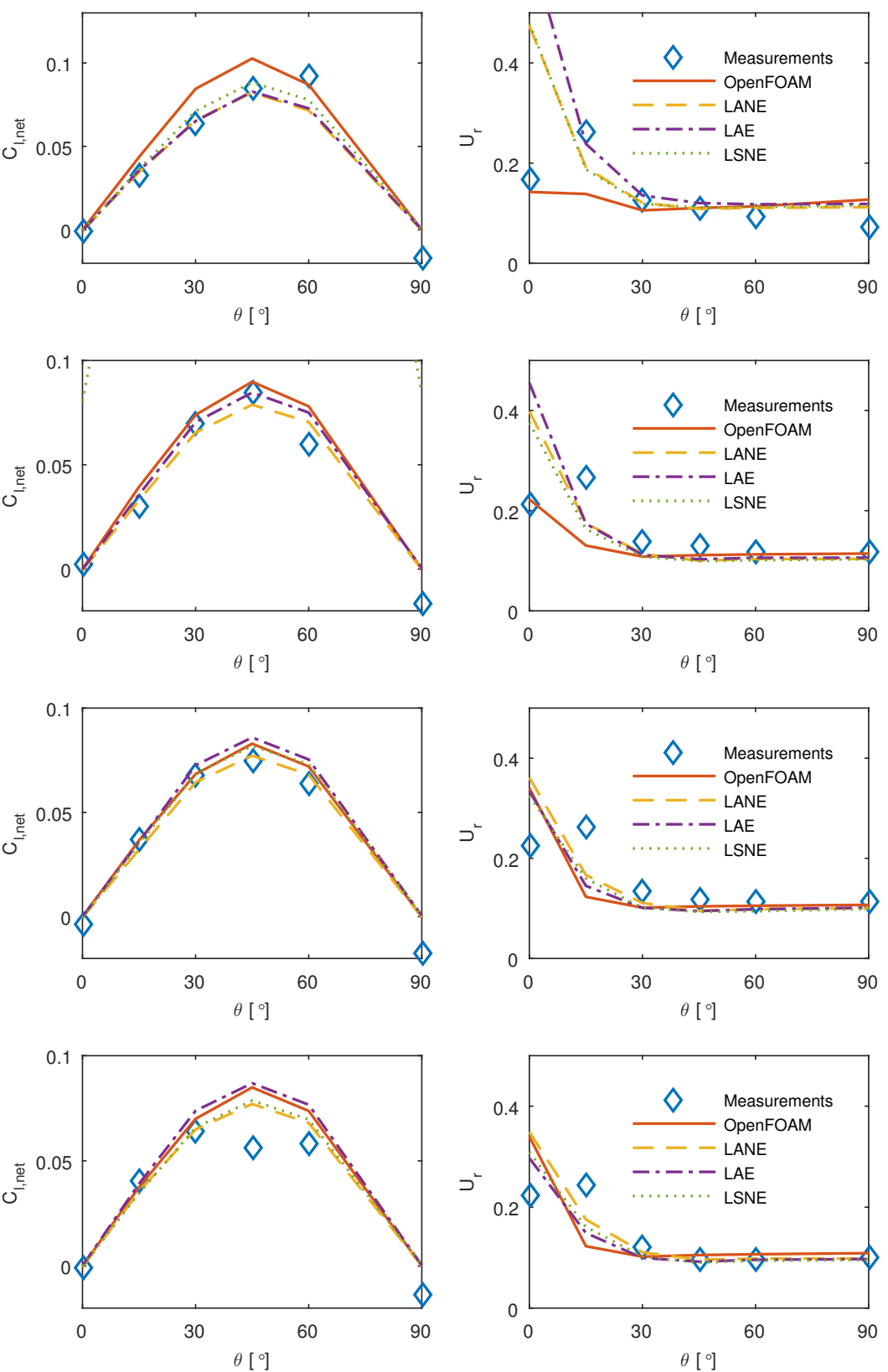

Figure 7: Comparison of the drag force coefficients $C_{d, n e t}$, lift force coefficients $C_{l, n e t}$ and velocity reduction factors $U_{r}$ from experimental data in Patursson (2007), the present numerical simulations in OpenFOAM, the fitted data based on a least square absolute normalized error (LANE), least square absolute error function(LAE), and least square normalized error function (LSNE) as shown in Patursson et al. (2010) for different incoming velocities: (a) $\mathrm{u}=0.125 \mathrm{~m} / \mathrm{s}$. (b) $\mathrm{u}=0.25 \mathrm{~m} / \mathrm{s}$, (c) $\mathrm{u}=0.5 \mathrm{~m} / \mathrm{s}$, (d) $\mathrm{u}=0.75 \mathrm{~m} / \mathrm{s}$. 

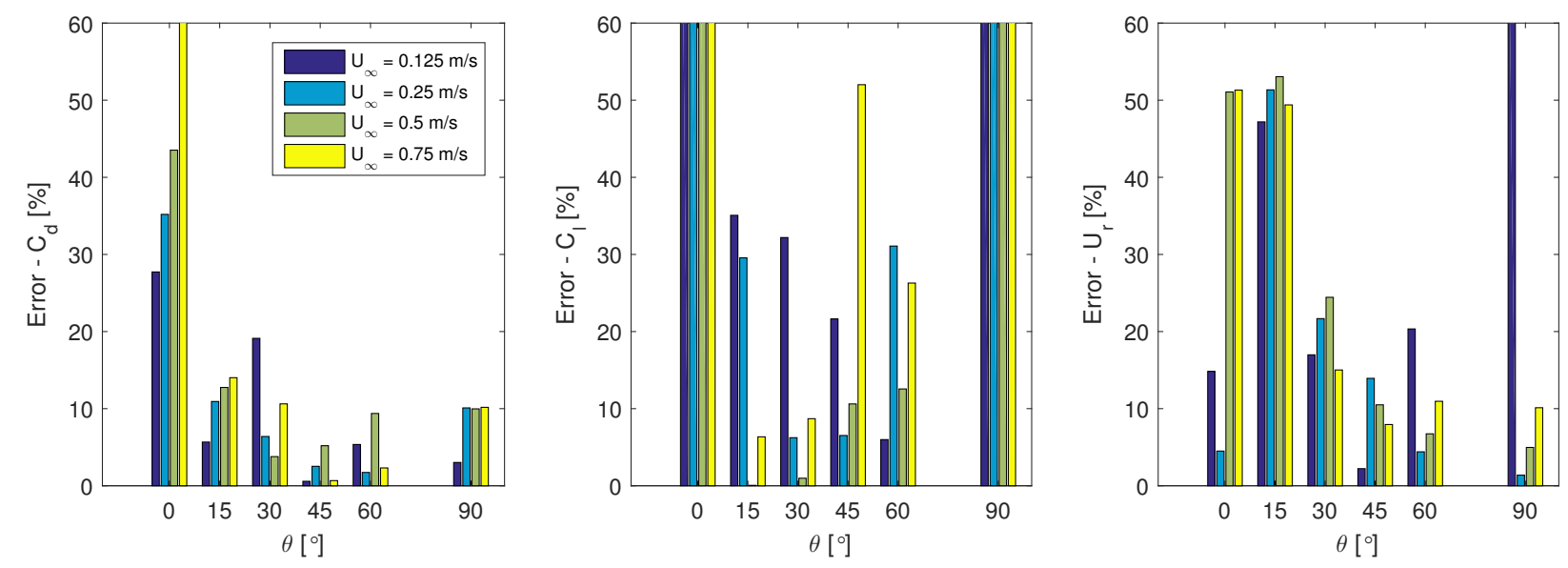

Figure 8: The relative error between numerical simulation and experimental data in Patursson et al. (2010).

The comparison between the numerical simulations and laboratory tests is presented in Fig. 10, and the relative error is given in Fig. 11. For net case 1, the relative errors are varying significantly with different attack angles and incoming velocities. The largest discrepancy between the numerical prediction and experimental data was for case with $\theta=30^{\circ}$ and $U_{\infty}=0.5 \mathrm{~m} / \mathrm{s}$, where the drag force was overpredicted about $40 \%$ by the numerical model. However, the relative errors for most of the remaining cases are well below $20 \%$. The drag force for net case 2 was well predicted by the numerical model, where the errors for most of the cases are about or below $10 \%$. The largest average error was associated with net case 3 . The drag force at all incoming velocities and attack angles were underestimated. This somehow indicated an underestimation of $a$ and $b$ for this net panel. For most cases the errors are between $10 \%-20 \%$. However, for cases with $U_{\infty}=0.75 \mathrm{~m} / \mathrm{s}$ and $U_{\infty}=1 \mathrm{~m} / \mathrm{s}$ at $\theta=30^{\circ}$, the relative errors reach $30 \%$.

\subsection{Current interaction with fixed circular fish cages}

Zhan et al. (2006) also conducted experiments on circular fish cage in steady current, and the numerical model was also validated against this set of experiments. The purpose is to examine the performance of the numerical model for more complex flows. The general setup of the experiments was the same with the experiments described in Section 6.2. The net used in the experiments are also exactly the same, and they were fixed tightly in a circular rigid frame well below the water surface.

The numerical model was set up in the same manner as described in Section 6.2. The sketch of the computational domain is given in Fig. 12. A local cylindrical coordinate was set up for the circular cage, where the origin of the coordinate was aligned with the center line of the circular fish cages. The porous resistance coefficients for the fish cages were exactly the same as shown in Table 3 in this local cylindrical coordinate system, and they were transormed to global coordinate system for each grid cell.

An example of flow visualization is presented in Fig. 13, where the velocity contours is plotted on the horizontal plane through the center of the circular fish cage with $S_{n}=0.128$. The incoming velocity is $U_{\infty}=0.5 \mathrm{~m} / \mathrm{s}$. In front of the fish cage, there exists a small area where the velocity was reduced. The main velocity reduction area is inside and behind the cage. The wake approximately has the same width with the diameter of the fish cage in the cross flow direction, which is the same with the flow characteristics described in Section 6.2 for plane net panel in current. The velocity inside the circular cage was reduced to approximately $0.96 U_{\infty}$. This is a reasonable value considering the relatively small solidity ratio of the net used in the simulation. Similar results were also reported in Bi et al. (2013b) and Løland (1993). Further reduction was also observed in the wake behind the cage due to the rear part of the cage.

Comparison of the drag force of the circular cages between numerical simulations and experimental data is presented in Fig. 14 and the associated relative errors are plotted in Fig. 15. The overall characteristics of the comparison are the same with the plane net panel in Section 6.2. For net case 1 the significant variation 


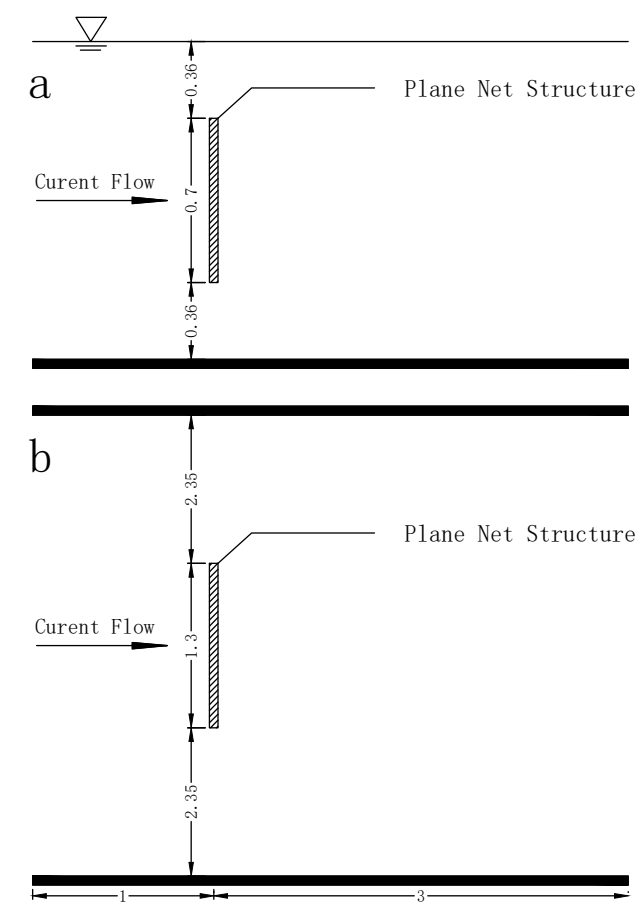

Figure 9: Sketch of the domain for reproduction of the experiments for current interaction with plane net panels in Zhan et al. (2006). (a) side view. (b) top view. (unit: $\mathrm{m}$ )

Table 4: The parameters of the regular waves from Lader et al. 2007a) and Lader et al. 2007b)

\begin{tabular}{cccccc}
\hline Wave case no. & 1 & 2 & 3 & 4 & 5 \\
\hline Wave frequency, $f(\mathrm{~Hz})$ & 1.42 & 1.42 & 1.42 & 1.25 & 1.00 \\
Wave length, $L(\mathrm{~m})$ & 0.77 & 0.77 & 0.77 & 1.00 & 1.54 \\
Wave period, $T(\mathrm{~s})$ & 0.70 & 0.70 & 0.70 & 0.80 & 1.00 \\
Wave height, $H(\mathrm{~m})$ & 0.044 & 0.064 & 0.084 & 0.104 & 0.165 \\
\hline
\end{tabular}

occurs again for cases with different incoming velocities. But the maximum error was reduced to less than $30 \%$ for case with $U_{\infty}=0.25 \mathrm{~m} / \mathrm{s}$. The numerical model for net case 2 gave the best prediction, where for all the case the relative error was well below $10 \%$. Meanwhile the underprediction of drag force for net case 3 was also reflected here in the simulation, where for all three incoming velocities, the drag force for the circular cylindrical cage was underestimated by $20 \%$ approximately.

\subsection{Wave interaction with net panels}

In Lader et al. (2007a) and Lader et al. (2007b), series of experiments were performed for analysis of wave interaction with net panels in the hydrodynamic laboratory at the Department of Mathematics, University of Oslo. Net panels with $0.5 \mathrm{~m}$ wide and $1.0 \mathrm{~m}$ high were positioned in the $8.3 \mathrm{~m}$ downstream of the wave maker, and attached at the top and bottom by custom made net forks to hold the net in place. The flume was $25 \mathrm{~m}$ long, $0.5 \mathrm{~m}$ wide and $1.0 \mathrm{~m}$ high, and the still water depth was set to $0.62 \mathrm{~m}$. In the experiments three kinds of net panels were used, and they were exposed to five different regular wave conditions. The relevant wave parameters are given in Table 4. A piston type wave maker with a vertical flap was used to generate waves. Two wave gauges were installed in the upstream and downstream with a distance of $1.4 \mathrm{~m}$ from the net to measure the surface elevation. Readers are referred to Lader et al. (2007a) and Lader et al. (2007b) for detailed information on setup of the experiments. 
a

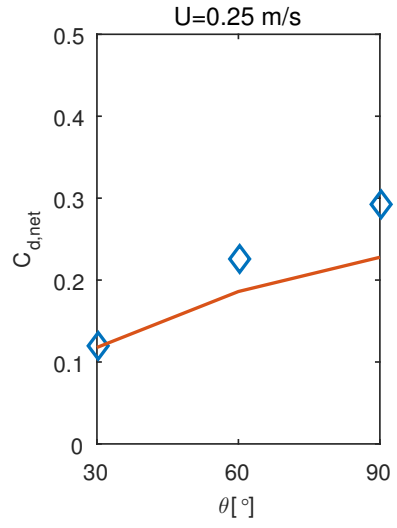

b

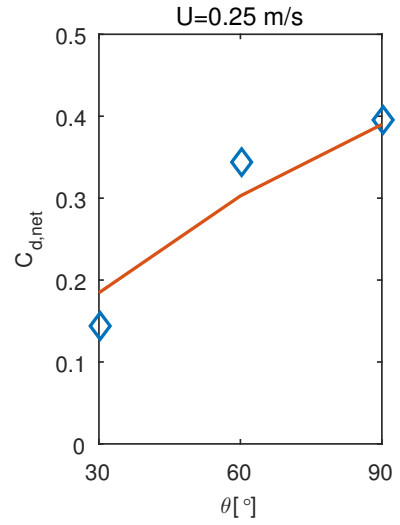

C

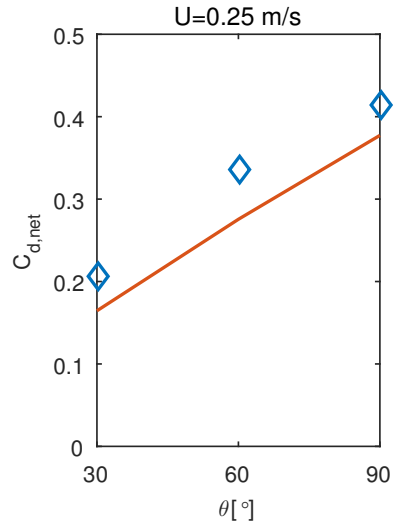

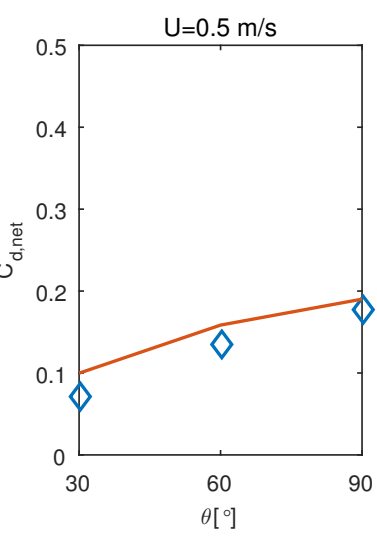
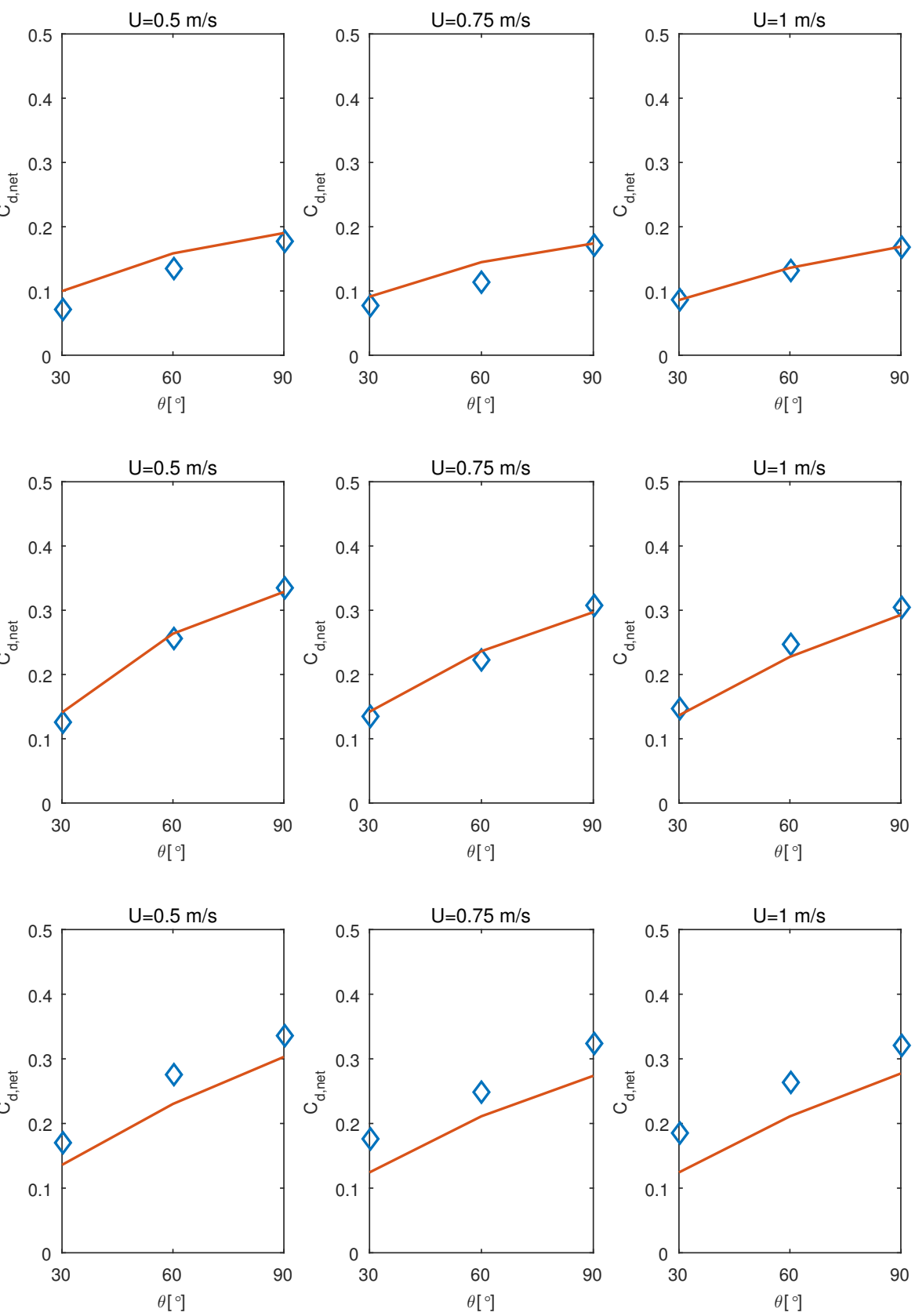

Figure 10: Comparison of the drag force coefficients $C_{d, n e t}$ between the present numerical simulations in OpenFOAM (solid line) and the data from laboratory tests in Zhan et al. (2006) (diamond) for three net panels: (a) net case 1 (b) net case 2 (c) net case 3 . 

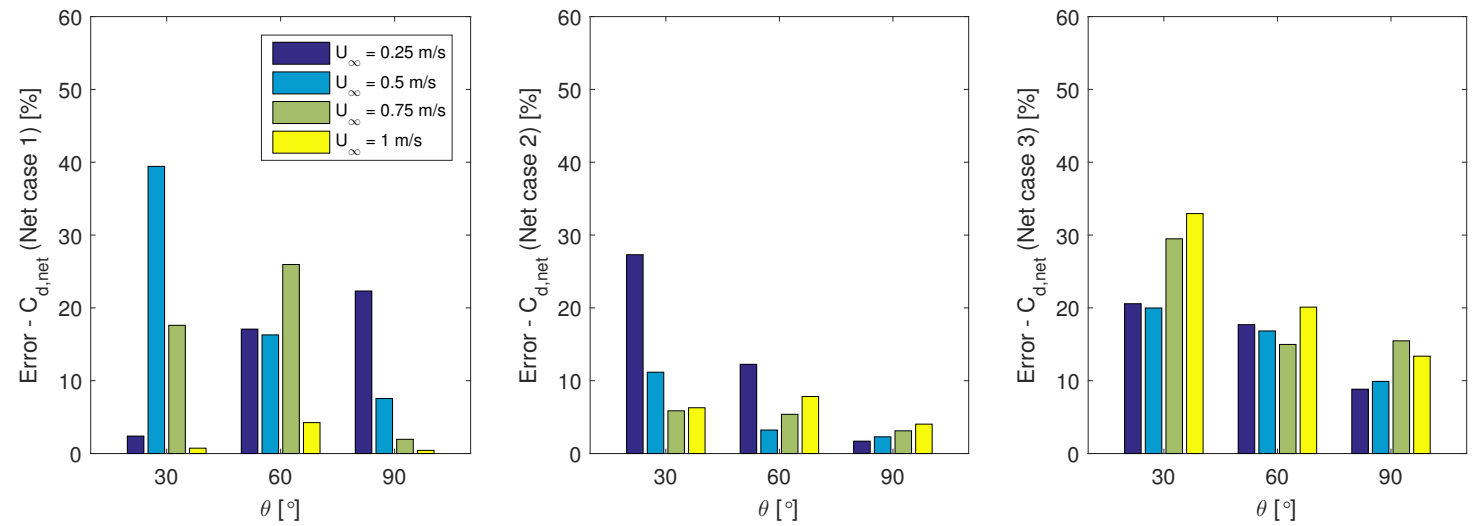

Figure 11: The relative error between numerical simulation and experimental data in Zhan et al. (2006) for plane net panels in steady current.

A two dimensional numerical wave tank was set up based on the physical experiments, since the width of the flume in the experiments was the same with the width of the net panels, and three dimensional effects could be neglected. The utility developed in Jacobsen et al. (2012) was applied for wave generation and absorption. Fig. 16 depicts the domain of the numerical wave tank where two relaxation zones were set up at the inlet and outlet of the numerical tank. In the center of wave tank the net panel was represented by a sheet of porous media with thickness of $50 \mathrm{~mm}$ and height of $1 \mathrm{~m}$, and the waves were generated according to the stream function wave theory in Fenton (1988). The calculated quadratic drag resistance coefficients of the three net panels are presented in Table 5, where the drag coefficients were estimated from Sumer and Fredsøe (2006).

Fig. 17- Fig. 21 depicts comparison of the time series of surface elevation, drag and lift forces between numerical simulations in OpenFOAM and experimental results from Lader et al. (2007a). Fig. 17(a) - Fig. 21(a) presents the surface elevation at the position of net when the wave was propagating without net. It was seen that the higher harmonic components in the wave motion were captured accurately by the numerical simulation and the surface elevation of the generated waves in the numerical model agreed well with the experimental results.

When analyzing the experimental results of wave forces on the net panels, higher harmonic components and multiple extreme points were observed within each zero crossing interval, e.g. in the crest of force cycles in Fig. 19 (g). In Lader et al. (2007a) the introduction/increase of the high harmonic components was explained as a result of the nonlinearity in the wave to force process: since the wave force is dependent on not only particle velocity but also exposed surface area, therefore the time series of the wave force should contain higher order components than wave motion itself. Due to the higher harmonics in the force waveforms, multiple extreme points also exists in each zero crossing interval. However, although in the numerical model, the forces were integrated at the instantaneous wet volume of the porous media, the multiple extreme points were not able to be captured, and only single extreme point occurs within each zero crossing interval. It was believed that the occurrence of the multiple extreme points was not only due to the higher harmonic components, but also due to the complex flow interaction between twines and knots, and the vortex shedding behind the twines. The lack of vortex shedding can also partially explain the smoother curves from the numerical simulation compared to the experiments. With such high KC number, the shedding frequency may increase which results in high frequency oscillations. Furthermore, in model scale experiments, the mesh bar length is comparable with the wave height. Therefore the significant variation of instantaneous wet volume might be another reason of this oscillation.

The phase shift is another feature worth mentioning. In general from the experiments it was observed that the horizontal force is approximately in phase with the wave elevation. However for some of the cases a slight shift relative to the wave phase was observed, e.g. the crest of the force signal in Fig. 18 (f). This phenomenon was not captured by the numerical model, and the horizontal forces from the numerical 


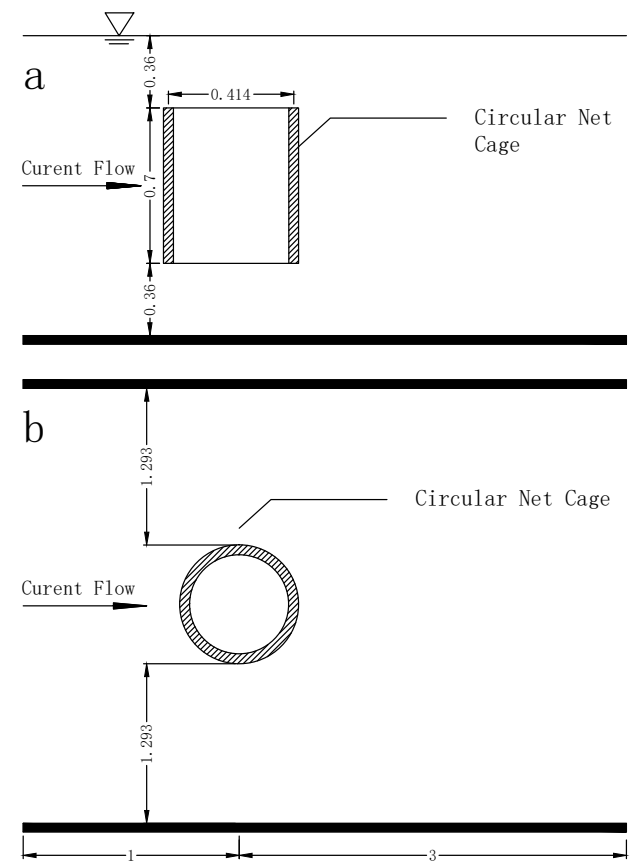

Figure 12: Sketch of the domain for reproduction of the experiments for current interaction with circular fish cages in Zhan et al. (2006). (a) side view. (b) top view. (unit: m)

simulations are in phase with the surface elevation.

Fig. 22 depicts the relative error between the prediected and measured wave height for different cases. The relative error was defined as the average of the ratio between the absolute error of the wave height and the wave height from experimental data. The absolute error includes error from both wave peak and wave trough. By this definition the shift up or down from the numerical results (e.g. the numerical results in Fig. 17(b)) was also accounted for in the error definition. It was found in Fig. 22 that for the first three wave conditions, the relative errors are in-between $20 \%-40 \%$ for most of the cases. However, for wave condition 4 and 5, most of the relative errors are reduced to about $20 \%$, except the drag force of net case 2 which suffers from a significant overprediction. We notice that in the experiments, the drag force of net case 2 under wave condition 4 and 5 has almost the same amplitude with drag force of net case 1 . This is difficult to explain, since under the same wave condition, the net with higher solidity ratio was expected to be subjected to larger wave load. This has been reflected in the first three wave conditions. Therefore there might be an underestimation to some degree from the experimental data. Meanwhile, the significant overprediction of the wave force from the numerical model may be partially due to the error in estimation of $C_{d, t w i n e}$. This is the motivation of the sensitivity analysis that will be presented in Section 7 In addition, there are some known issues in wave-making by CFD methods (especially OpenFOAM), this has been given in e.g. Wroniszewski et al. (2014). The near-surface kinematics were not able to be reproduced correctly by the solver, which might serve as an error source. Overprediction of the velocity close to the free surface will lead to the overprediction of wave forces. However this seems to be case sensitive, since for the first three wave conditions they are within the reasonable error bound.

\section{Sensitivity analysis on porous resistance coefficients}

The sensitivity analysis was carried out to examine if the results were still located in a reasonable bound when taking uncertainties of the numerical model into account. The overall procedure of the analysis is the same as shown in Kristiansen and Faltinsen (2012), where one of the selected parameters was varied while the 


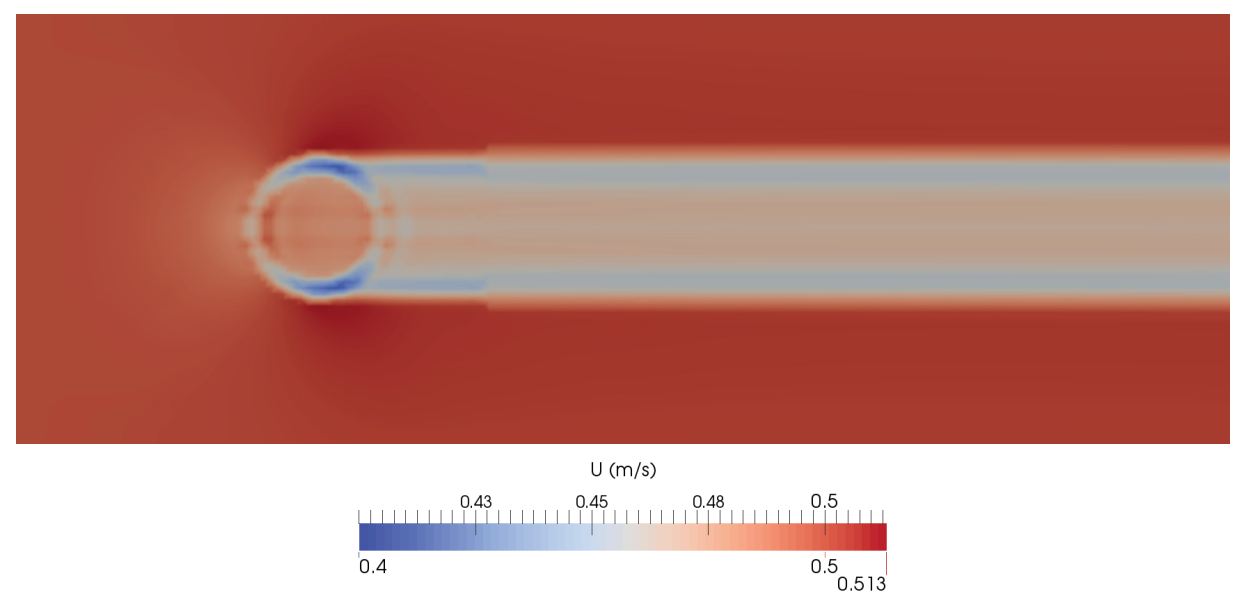

Figure 13: The velocity contours from CFD simulation on a horizontal plane cut through the center of the circular fish cage. The solidity ratio $S_{n}=0.128$ (net case 1 ). The incoming velocity is $0.5 \mathrm{~m} / \mathrm{s}$.
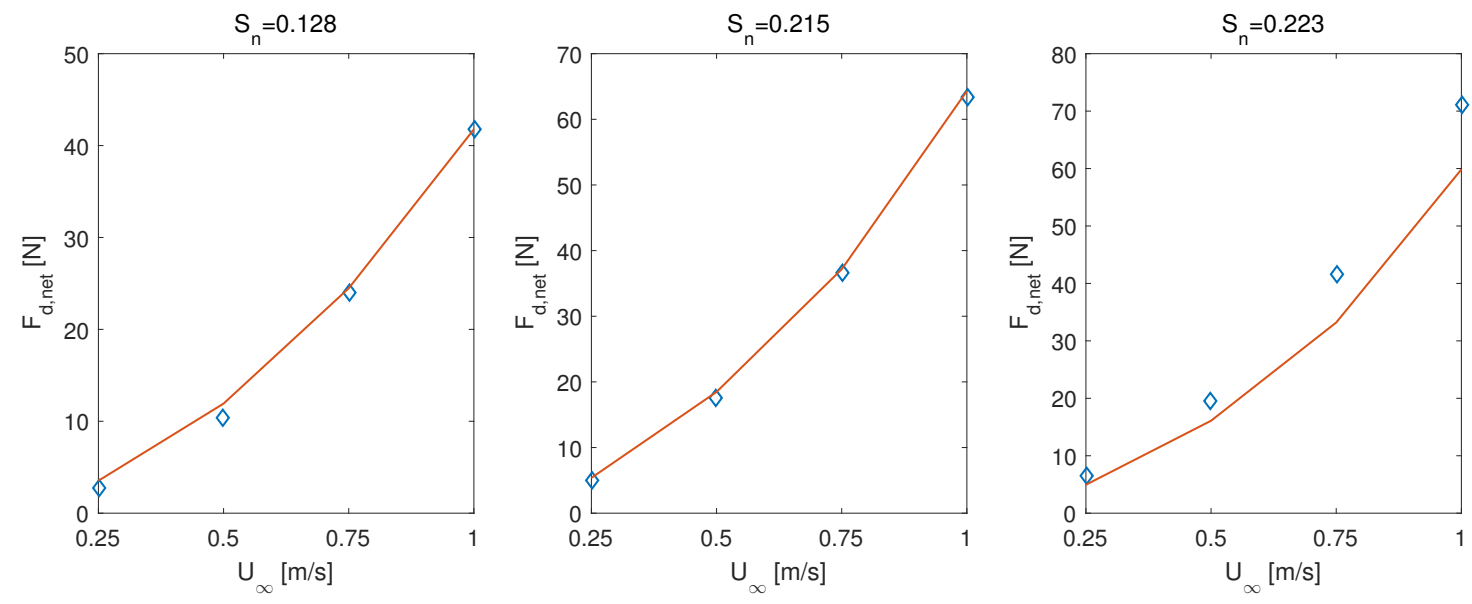

Figure 14: Comparison of the drag force $F_{d \text { net }}$ between the present numerical simulations in OpenFOAM (solid line) and the data from laboratory tests in Zhan et al. (2006) (diamond) for three circular fish cages.

others were kept the same as nominal values. However, in the present work we focused on the uncertainties in calculation of porous resistance coefficients, other uncertainties were not involved in the analysis. The uncertainties of the porous resistance coefficients come from the followings: The drag force coefficient of the twines for a fishing net was assigned with a $10 \%$ uncertainty due to i.e. misreading of the figure for drag force coefficients, difference between the shape of the real twine and a cylinder etc.; The projected area $S_{1}$ and $S_{2}$ for the in-plane and out-of-plane twines were varied with $5 \%$, since they were usually calculated based on the mesh distance $\lambda$ and the overall dimension of the fishing nets, therefore there exists round-off errors. The other parameters in Eq. (30-32) were usually given, therefore uncertainties were not assigned on these values.

The total error bound $\Delta F$ of the uncertainties was estimated as:

$$
\Delta F=\left(\sum_{i}\left(\Delta F_{i}\right)^{2}\right)^{1 / 2}
$$

where $\Delta F_{i}$ is the error bound due to variation of each parameter. Eq. 49 indicates that the error sources 


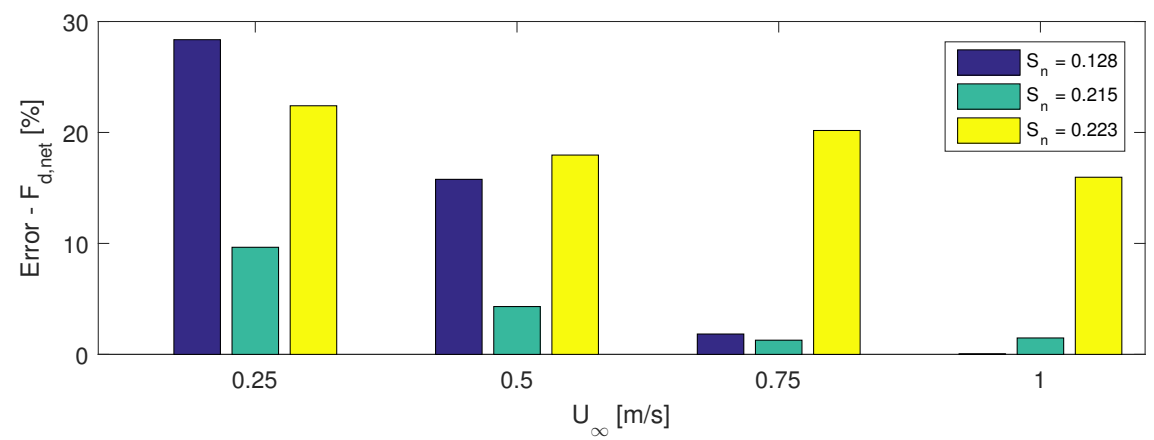

Figure 15: The relative error between numerical simulation and experimental data in Zhan et al. (2006) for three circular fish cages in steady current.

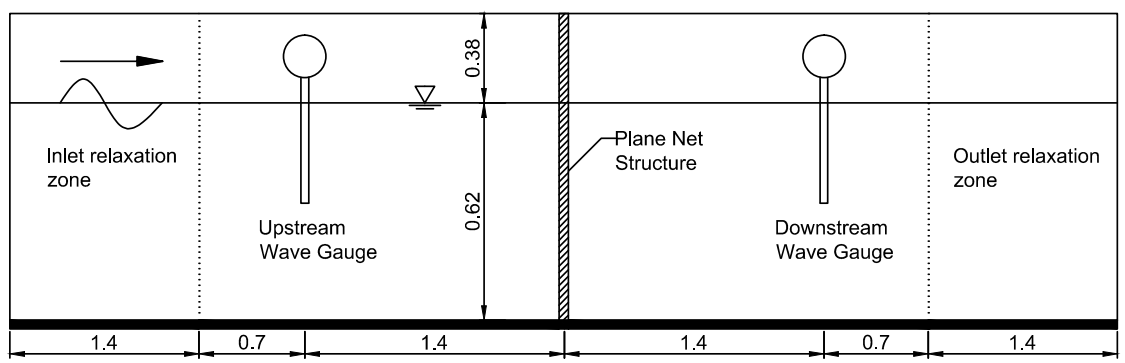

Figure 16: Sketch of the computational domain for simulation of wave interaction with plane net panel. (unit: m)

were assumed to be stochastically independent of each other. $\Delta F_{i}$ was calculated as:

$$
\Delta F_{i}=\frac{1}{2} \sum_{j=1}^{2}\left|F_{j}-F_{0}\right|
$$

where $F_{0}$ is the nominal force and $F_{j}$ is the force from a run with variation.

The results is shown in Fig. 23 for current flow through circular fish cages in Zhan et al. (2006). The case setup has been illustrated in detail in in Section 6.3. The following conclusion were drawn from the presented results: (1) In general solidity ratio has minor effect on the relative error of drag force due to uncertainties of the parameters, i.e. the circular cages with different solidity ratios have the same order of magnitude of error when one specific parameter was varied. (2) The incoming velocity has minor effect on the relative error of drag force, i.e. for a given fish cage, the relative errors of drag force due to variation of one specific parameter with different incoming velocities are in the same order of magnitude. (3) The relative error induced by uncertainties of $S_{1}$ and $S_{2}$ is insignificant, usually below 5\%. (4) The dominant error source is the uncertainty due to $C_{d, \text { twine. }}$. However, it was found that by $10 \%$ variation of drag force coefficient, the relative error of drag force in general is around or less than $10 \%$. We believe that this is an reasonable error bound. This is important or fishing nets in waves, where $C_{d, t w i n e}$ is difficult to find due to relatively small Re number but large $K C$ number. A rough estimation of $C_{d, t w i n e}$ in this case might still produce acceptable results.

\section{Conclusions}

The present paper investigates the porous media model with application to flow through fishing net structures, where the main effort was paid to derive new expressions for the porous resistance coefficients of the fishing net. The volume averaged Reynolds averaged Navier-Stokes equations were employed as the governing equations, and the differences of the mathematical formulations used in the present work and 
(a)

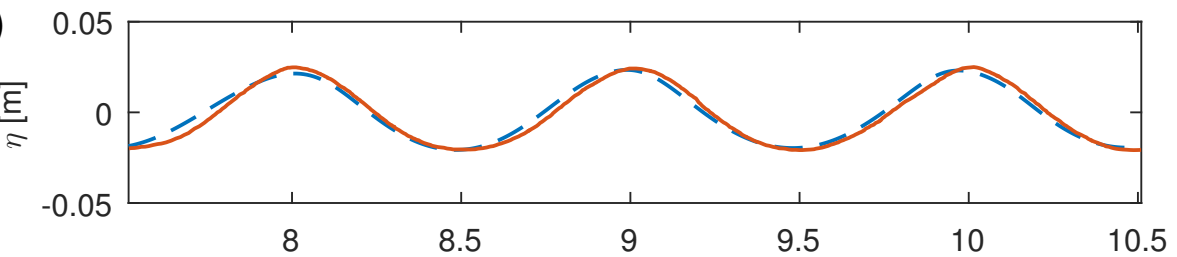

(b)

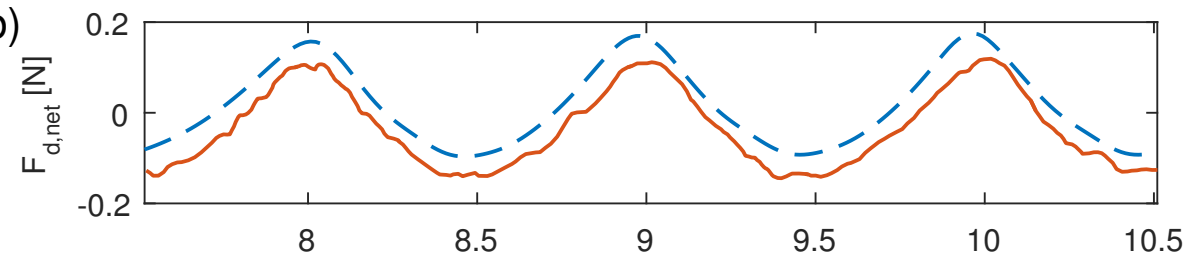

(c)

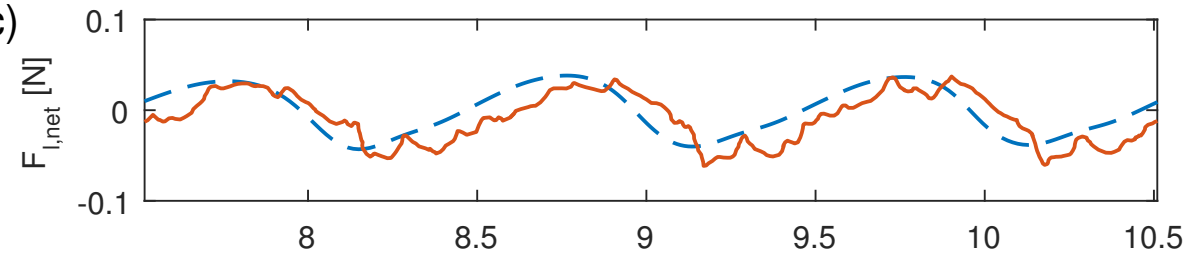

(d)

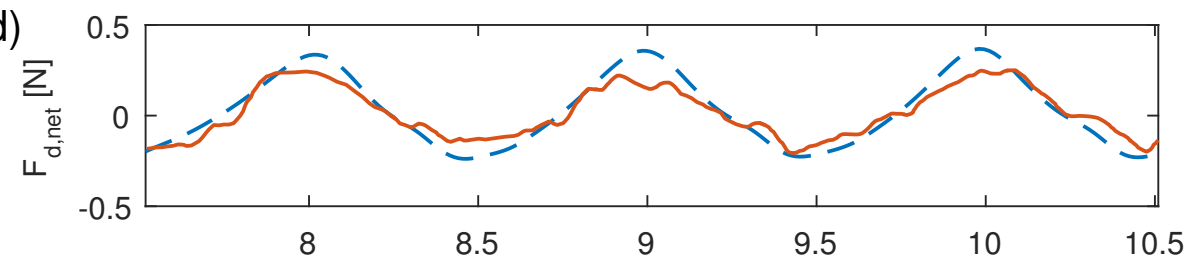

(e)

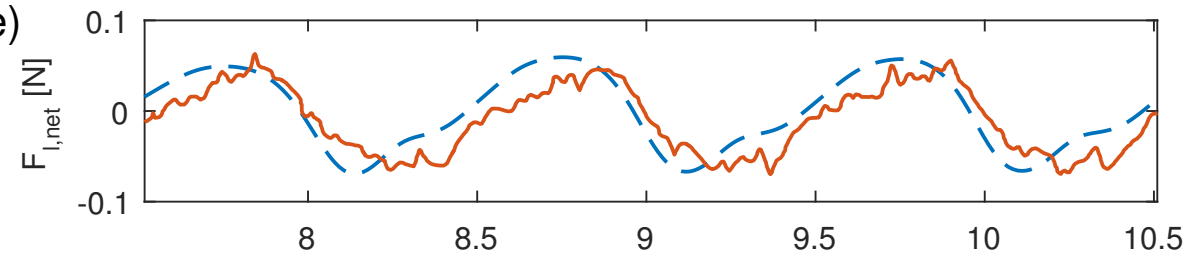

(f)
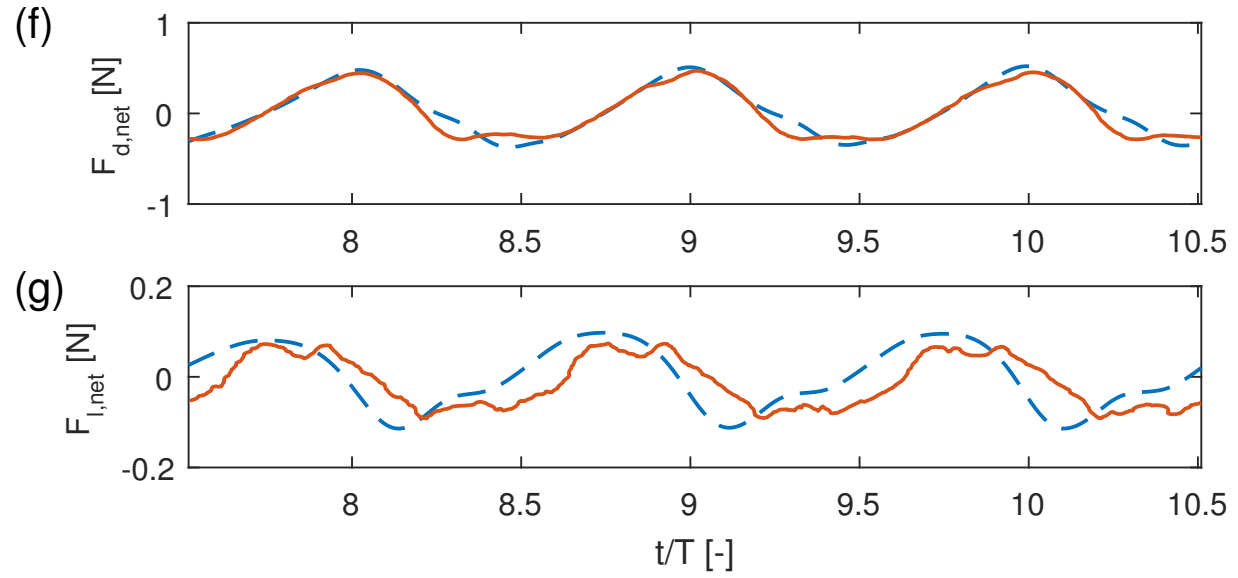

Figure 17: Comparison of time series of surface elevation, drag and lift forces between numerical simulation in OpenFOAM (dashed line) and experiments from Lader et al. (2007a) (solid line) for wave case 1 (wave frequency $f=1.42 \mathrm{~Hz}$, wave height $H=0.044 \mathrm{~cm}$ ). (a) surface elevation at the position of the net when wave propagating without net. (b) drag force for net case 1. (c) lift force for net case 1. (d) drag force for net case 2. (e) lift force for net case 2. (f) drag force for net case 3. (g) lift force for net case 3 . 

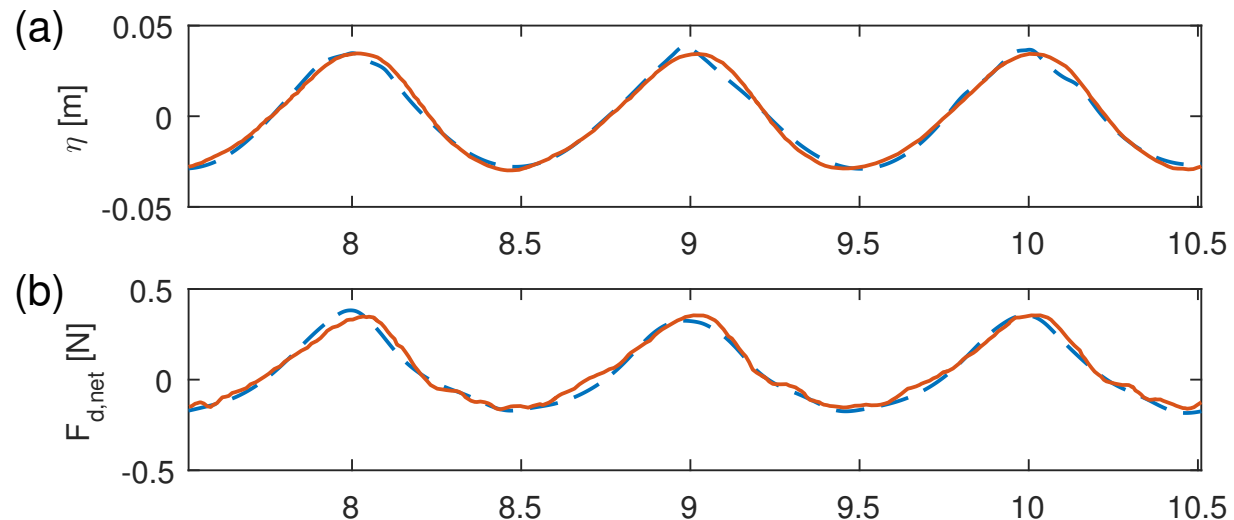

(c)

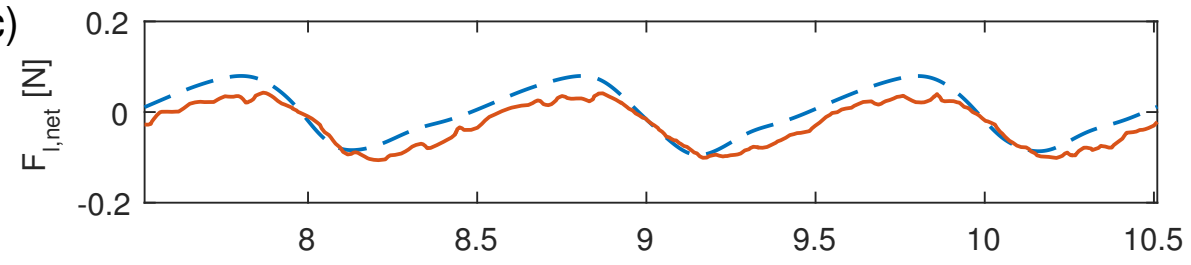

(d)

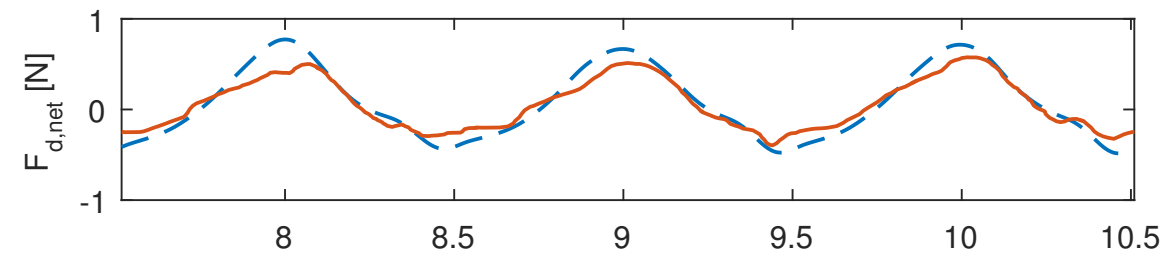

(e)

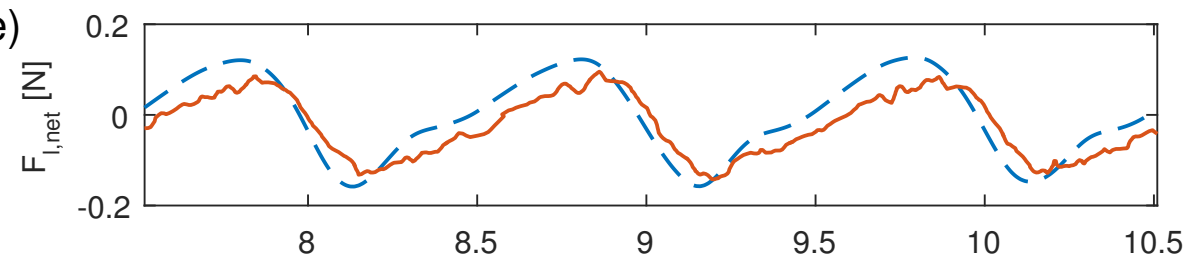

(f)
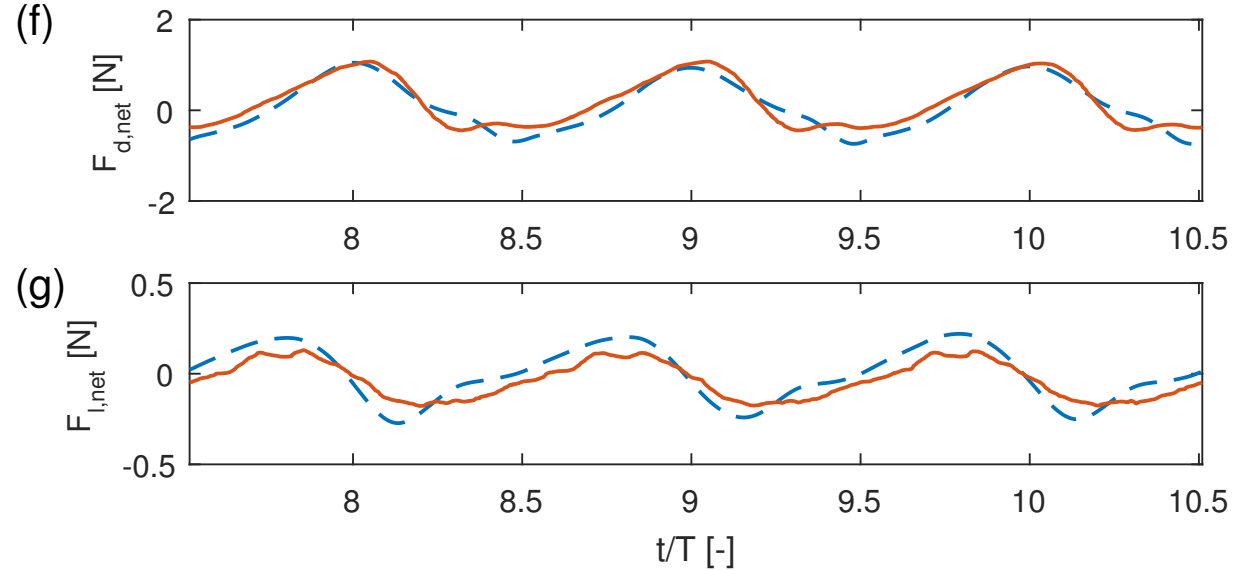

Figure 18: Comparison of time series of surface elevation, drag and lift forces between numerical simulation in OpenFOAM (dashed line) and experiments from Lader et al. (2007a) (solid line) for wave case 2 (wave frequency $f=1.42 \mathrm{~Hz}$, wave height $H=0.064 \mathrm{~cm})$. Legend as the same with Fig. 17 
(a)

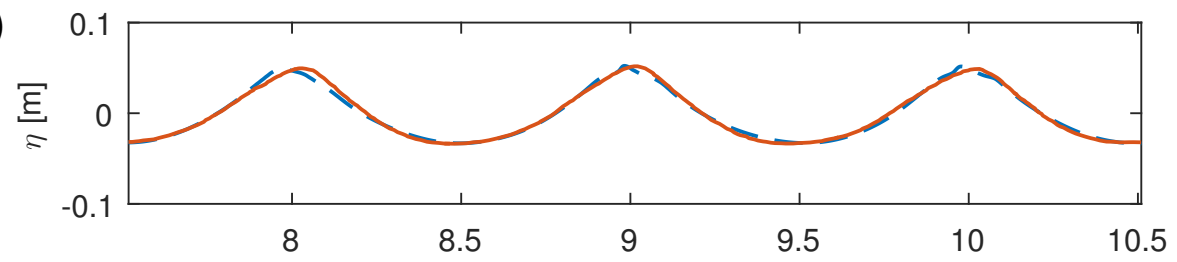

(b)

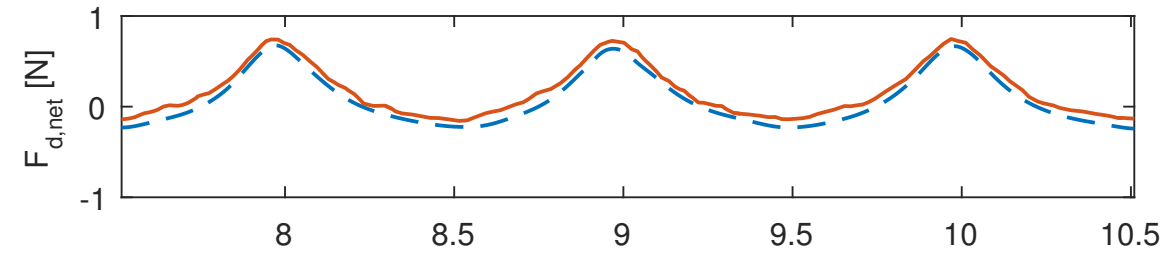

(c)

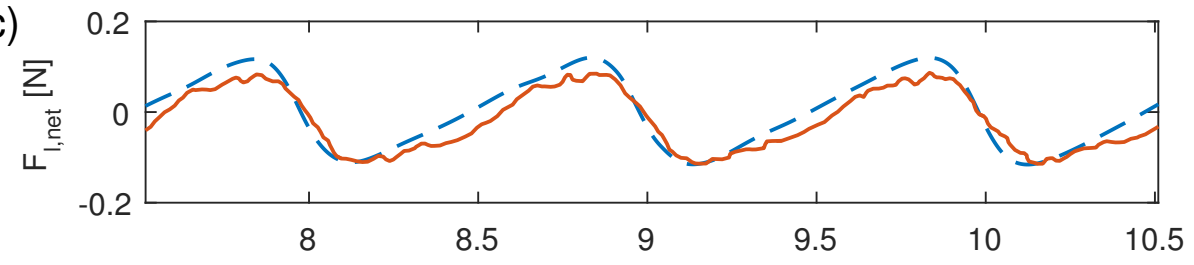

(d)

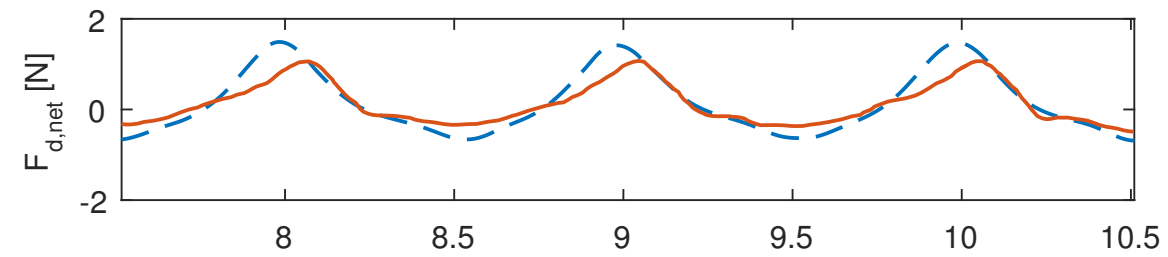

(e)

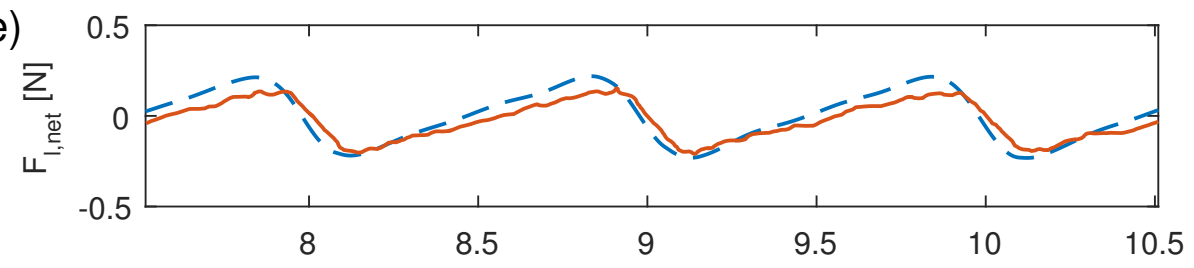

(f)

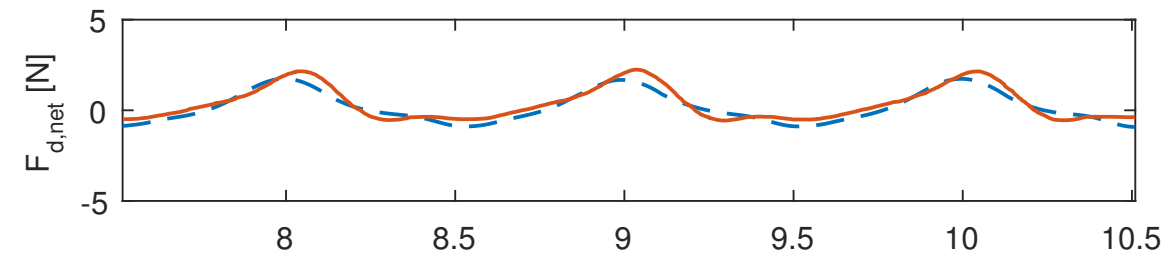

(g)

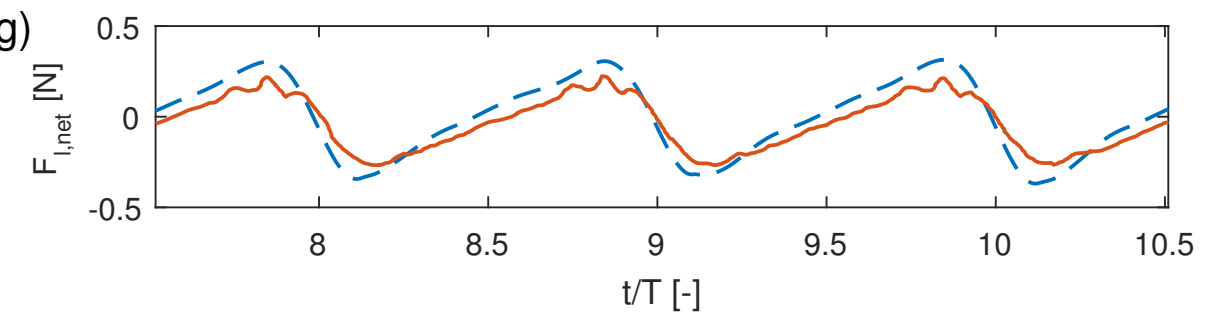

Figure 19: Comparison of time series of surface elevation, drag and lift forces between numerical simulation in OpenFOAM (dashed line) and experiments from Lader et al. (2007a) (solid line) for wave case 3 (wave frequency $f=1.42 \mathrm{~Hz}$, wave height $H=0.084 \mathrm{~cm}$ ). Legend as the same with Fig. 17 
(a)

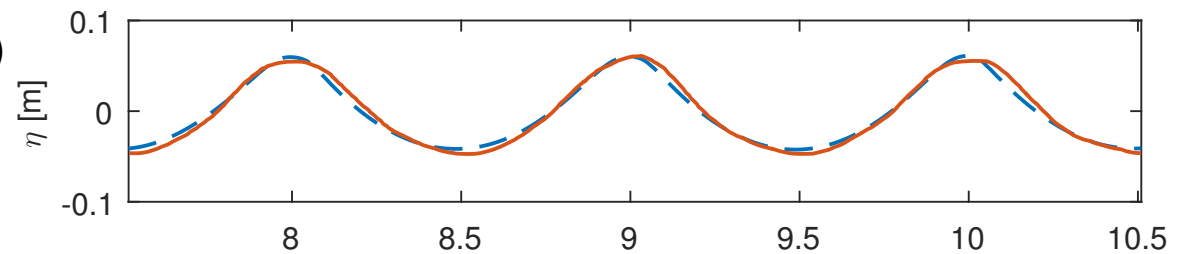

(b)

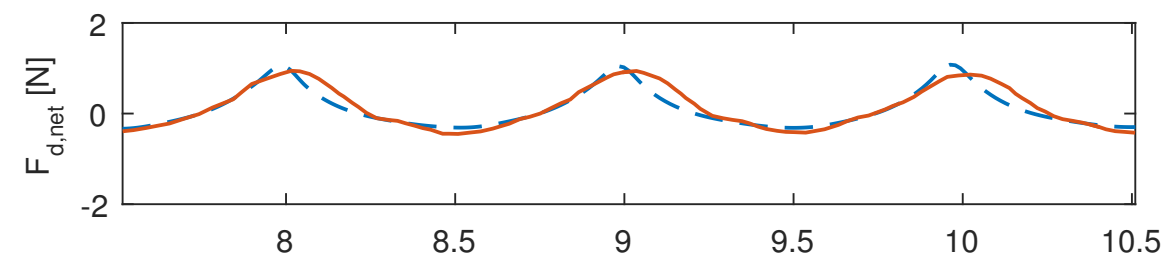

(c)

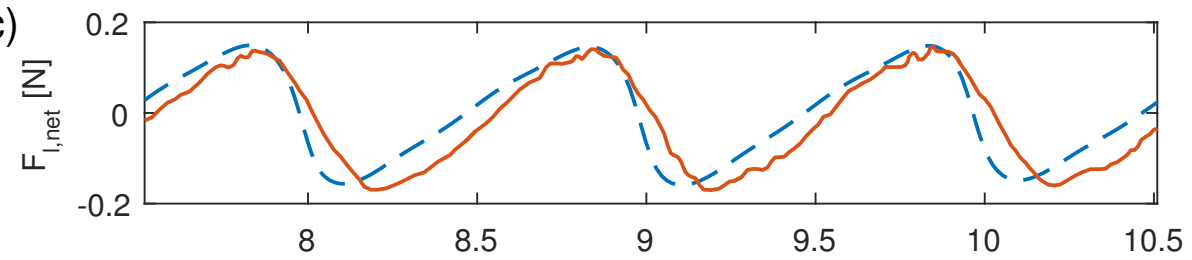

(d)

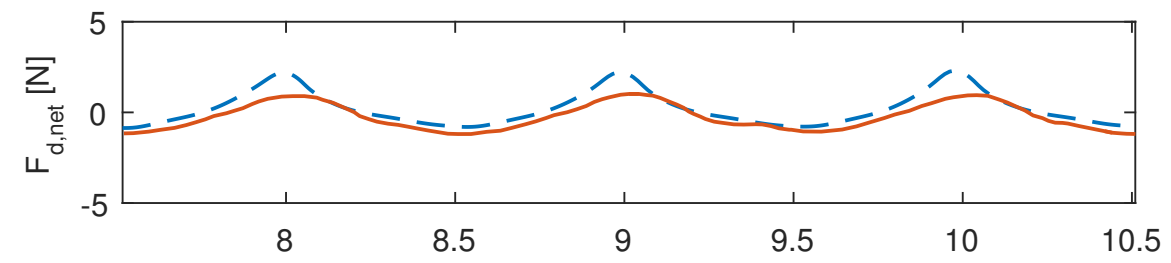

(e)

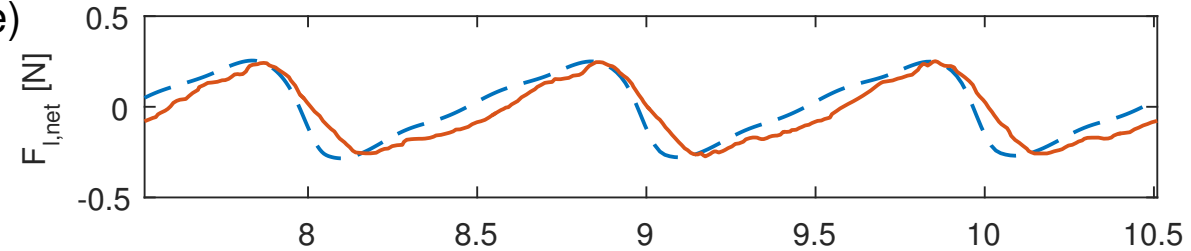

(f)
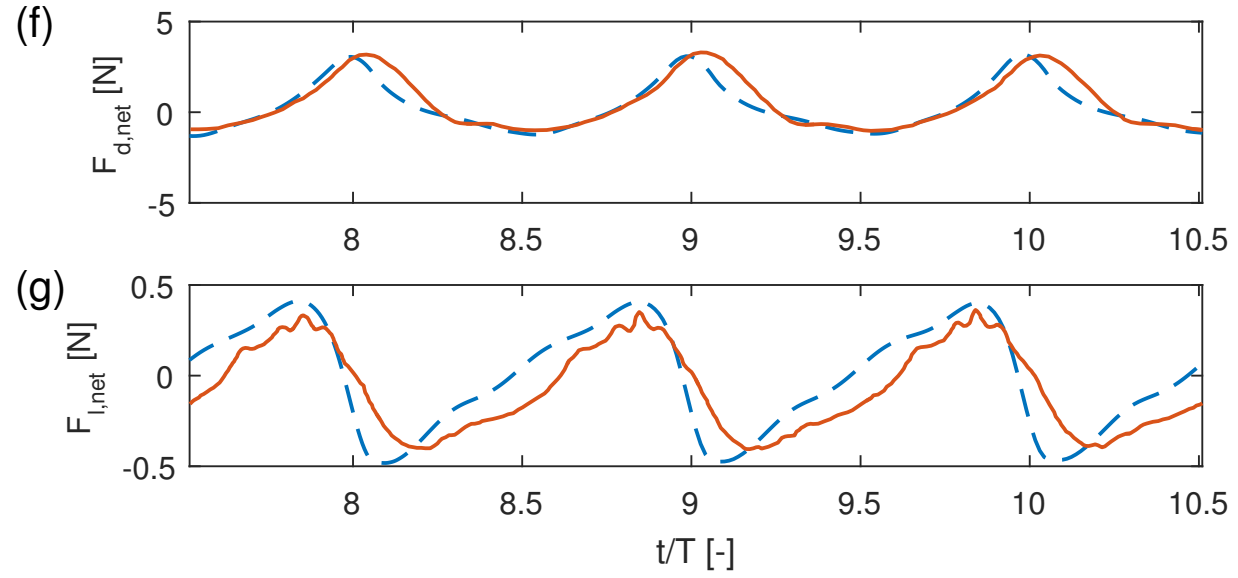

Figure 20: Comparison of time series of surface elevation, drag and lift forces between numerical simulation in OpenFOAM (dashed line) and experiments from Lader et al. (2007a) (solid line) for wave case 4 (wave frequency $f=1.25 \mathrm{~Hz}$, wave height $H=0.104 \mathrm{~cm})$. Legend as the same with Fig. 17 
(a)

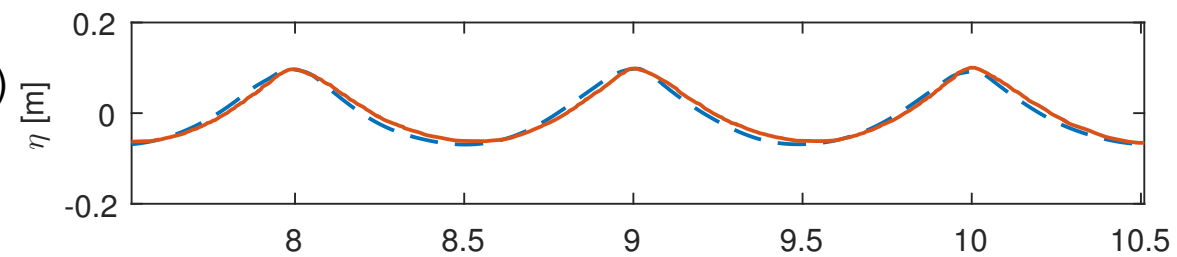

(b)

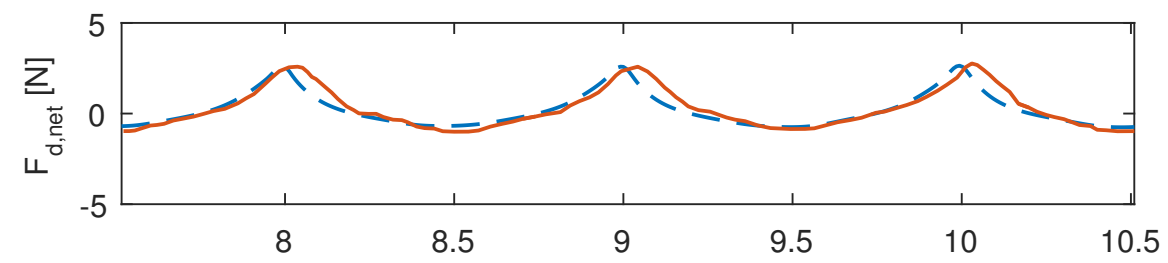

(c) $z$

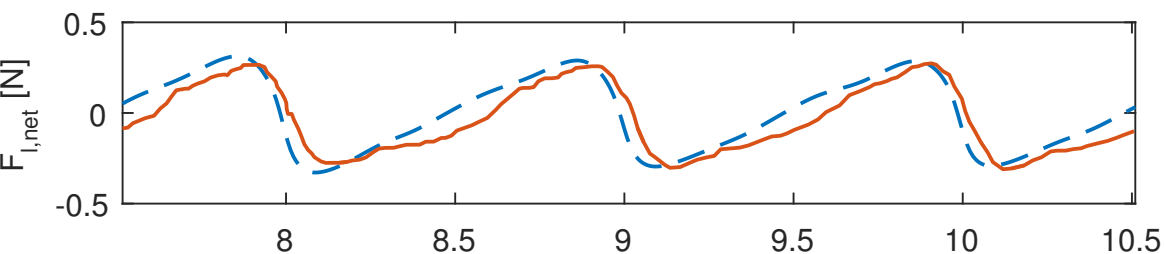

(d)

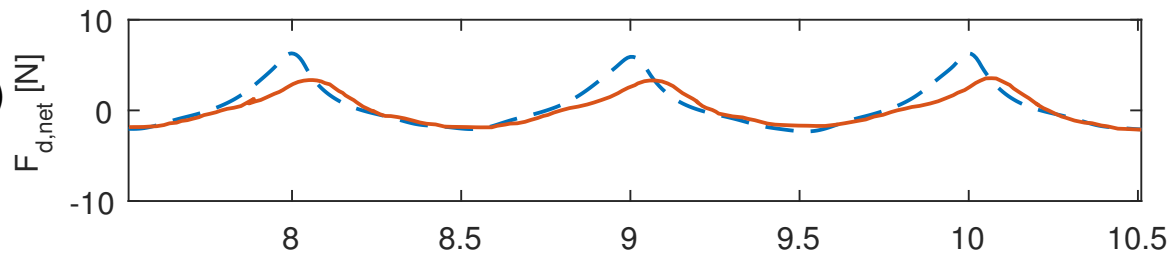

(e)
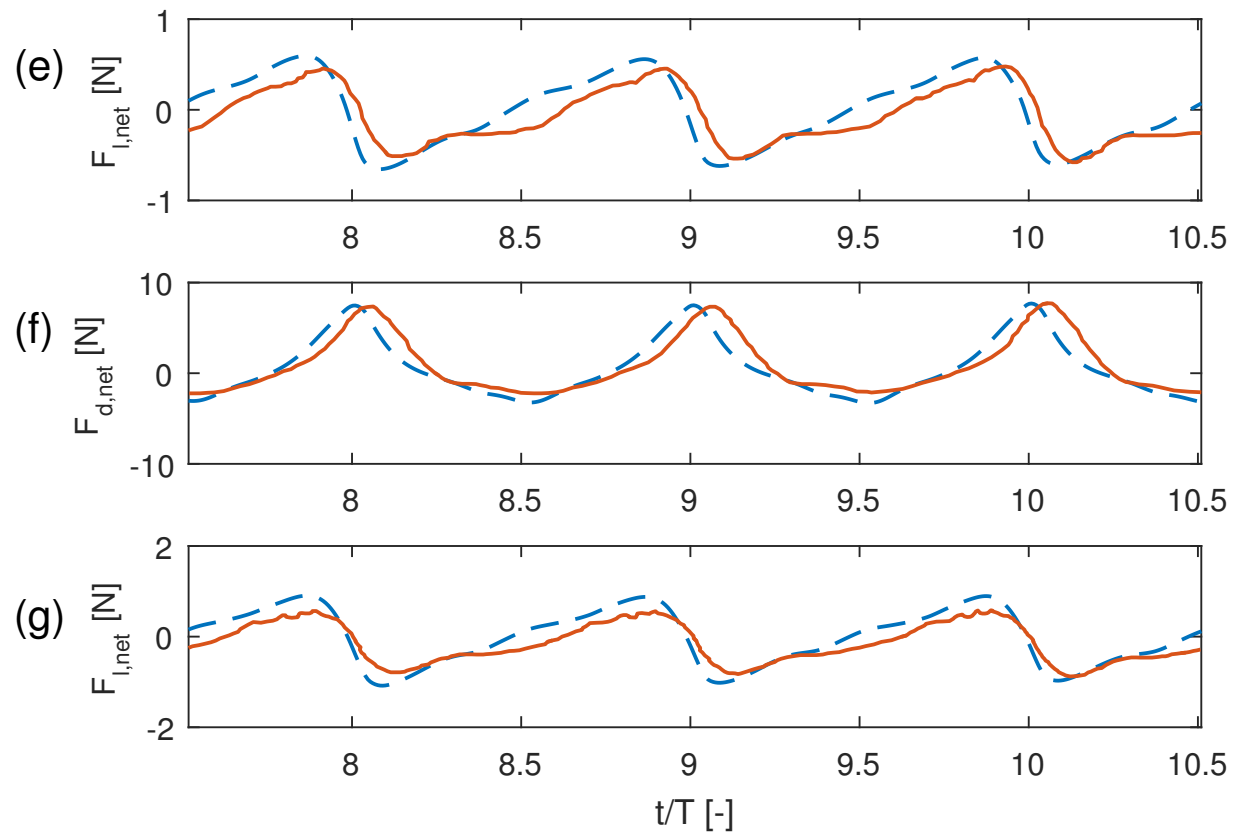

Figure 21: Comparison of time series of surface elevation, drag and lift forces between numerical simulation in OpenFOAM (dashed line) and experiments from Lader et al. (2007a) (solid line) for wave case 5 (wave frequency $f=1.00 \mathrm{~Hz}$, wave height $H=0.165 \mathrm{~cm})$. Legend as the same with Fig. 17 
Table 5: The physical parameters of the three nets used in Lader et al. (2007a) and Lader et al. (2007b), with calculation of the porous resistance coefficients in five wave conditions. The detailed wave conditions are given in Table 4

\begin{tabular}{|c|c|c|c|c|}
\hline Net case no. & & 1 & 2 & 3 \\
\hline$S_{n}$ & & 0.095 & 0.220 & 0.288 \\
\hline$\lambda(\mathrm{m})$ & & 0.021 & 0.016 & 0.025 \\
\hline$d(\mathrm{~m})$ & & 0.0010 & 0.0018 & 0.0036 \\
\hline$S_{1}\left(\mathrm{~m}^{2}\right)$ & & 0.0247 & 0.0571 & 0.0738 \\
\hline$S_{2}\left(\mathrm{~m}^{2}\right)$ & & 0.0252 & 0.0581 & 0.0756 \\
\hline$V\left(\mathrm{~m}^{3}\right)$ & & 0.0250 & 0.0250 & 0.0250 \\
\hline$a$ & & 1.22 & 1.42 & 1.69 \\
\hline$b$ & & 0.92 & 0.87 & 1.25 \\
\hline \multirow{5}{*}{ Wave case 1} & $\mathrm{Re}$ & 197 & 355 & 710 \\
\hline & $K C$ & 139 & 77 & 38 \\
\hline & $C_{d, \text { twine }}$ & 1.80 & 1.60 & 1.60 \\
\hline & $C_{1}$ & 4.63 & 11.15 & 15.17 \\
\hline & $C_{2}$ & 1.73 & 3.37 & 5.52 \\
\hline \multirow{5}{*}{ Wave case 2} & Re & 287 & 516 & 1033 \\
\hline & $K C$ & 202 & 112 & 56 \\
\hline & $C_{d, \text { twine }}$ & 1.70 & 1.50 & 1.50 \\
\hline & $C_{1}$ & 4.39 & 10.49 & 13.15 \\
\hline & $C_{2}$ & 1.64 & 3.17 & 4.78 \\
\hline \multirow{5}{*}{ Wave case 3} & $\mathrm{Re}$ & 377 & 678 & 1356 \\
\hline & $K C$ & 265 & 147 & 73 \\
\hline & $C_{d, \text { twine }}$ & 1.50 & 1.40 & 1.30 \\
\hline & $C_{1}$ & 4.15 & 9.84 & 13.15 \\
\hline & $C_{2}$ & 1.55 & 2.97 & 4.78 \\
\hline \multirow{5}{*}{ Wave case 4} & $\mathrm{Re}$ & 410 & 736 & 1472 \\
\hline & $K C$ & 305 & 169 & 85 \\
\hline & $C_{d, \text { twine }}$ & 1.40 & 1.30 & 1.30 \\
\hline & $C_{1}$ & 3.91 & 9.18 & 13.15 \\
\hline & $C_{2}$ & 1.46 & 2.77 & 4.78 \\
\hline \multirow{5}{*}{ Wave case 5} & $\mathrm{Re}$ & 500 & 898 & 1796 \\
\hline & $K C$ & 499 & 277 & 139 \\
\hline & $C_{d, t w i n e}$ & 1.20 & 1.30 & 1.20 \\
\hline & $C_{1}$ & 3.17 & 7.87 & 12.14 \\
\hline & $C_{2}$ & 1.19 & 2.38 & 4.41 \\
\hline
\end{tabular}

previous works have been discussed. The force acting on the net was obtained by integrating the porous resistance force over the instantaneous wet volume of the porous media.

The linear drag force was neglected in the present work, and only quadratic drag force was accounted for for the porous media resistance. The explanations have been given in terms of porous media model and physical background for flow through fishing nets. A new formula was proposed to address the lack of method on calculation of the quadratic drag force coefficient. The formula was derived based on the transformation of Morison type load model, and it follows the principle that the force acting on the porous media zone should be equal to the force obtained from the Morison type load model. The angle dependence was dropped in the derivation of the formula, in order to get a constant coefficient in time and space domain. This is based on the previous works which gain some good results under this assumption. The interaction effects between twines were accounted for by two new parameters, and they were calibrated by minimizing the error between forces from experimental results and the derived formula. Three reference values of the parameters were obtained based on the available experimental data, and a linear interpolation was used to 

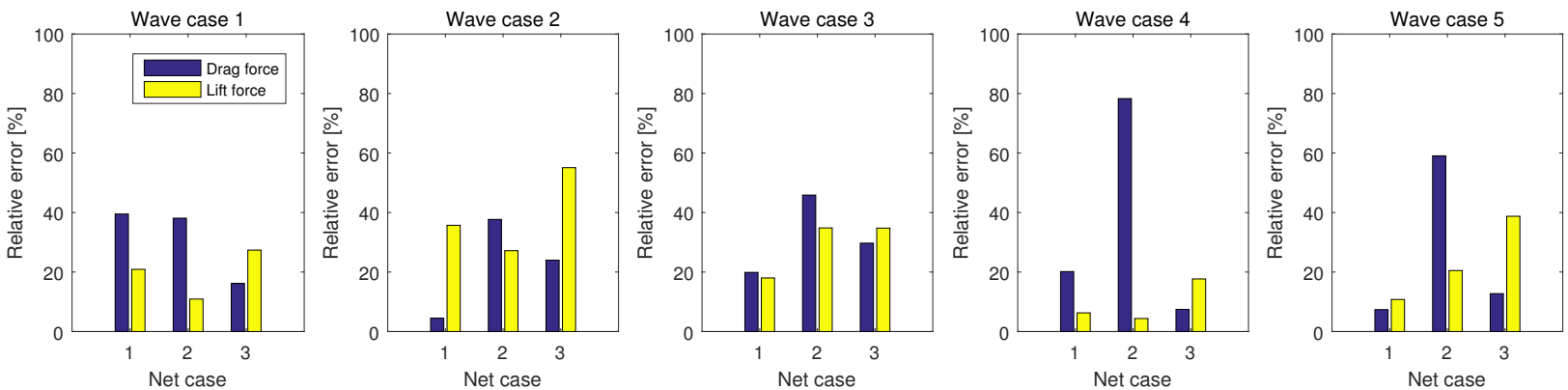

Figure 22: Relative error between results from the present numerical model and experimental data for the mean wave height in Lader et al. (2007a)

obtain the parameters for other nets with different solidity ratios.

The overall numerical model was extensively validated against available experimental data in the current literature. The validation includes both plane net panels and circular fish cages in both steady and unsteady flows. The comparison between numerical and experimental data was given for each validation case, and the relative error was also presented in percentage. It was found that for most of the cases the numerical model could reproduce the experiments adequately. The cases with large relative errors have been investigated and the reason has been explained.

Based on the overall performance, it was concluded that porous media model is a feasible approach for modeling flow through fishing net structures. The derived formula could give porous resistance coefficients of fishing nets within a reasonable error bound, indicating that $a$ and $b$ could account for the interaction effects to a large degree. However, regarding the assumption that angle dependence has minor effects on the numerical results, and application of time and space independent porous resistance coefficients is sufficient for modeling fish cage, more validations are necessary in more complex 3D flow scenarios, i.e. circular fish cages in wave or combined wave and current conditions.

\section{Acknowledgments}

We would like to acknowledge the reviewers for their comments and suggestions, which help us understand the problem in depth and improve the quality of the paper. Furthermore, Dr. Bjarne Jensen is acknowledged for his work on development of VARANS equations and porous media model in OpenFOAM, and the enlightening discussions on inertia effects of the porous media. The research was partially supported by FP7-OCEAN-2011 project "Innovative Multi-purpose offshore platforms: planning, design and operation", MERMAID, 288710, under the call "Ocean of Tomorrow".

\section{Appendix A. Comparison of the two coordinate transformation approaches}

When modeling flow through fishing nets by porous media model, the anisotropy property must be taken into account since the porous resistance is influenced by the orientation of the porous media. They must be transformed from local to global coordinate system before calculating the resistance forces.

In Patursson et al. (2010) and Zhao et al. (2013b), the porous resistance force was modeled by DarcyForchheimer equation as shown in Eq. (9). But two different approaches were applied in the transformation of the porous resistance coefficients. In this section analysis will be given on the differences and limitations of the approaches.

For the most universal cases where neither the flow direction nor the local coordinate of the porous media is aligned with the global coordinate, a strict $3 \mathrm{D}$ coordinate transformation matrix $R$ must be employed. Therefore $D$ and $C$ in global coordinate system were formulated as:

$$
D_{i j}=R_{i p} R_{j q} D_{p q}^{*} \quad C_{i j}=R_{i p} R_{j q} C_{p q}^{*}
$$



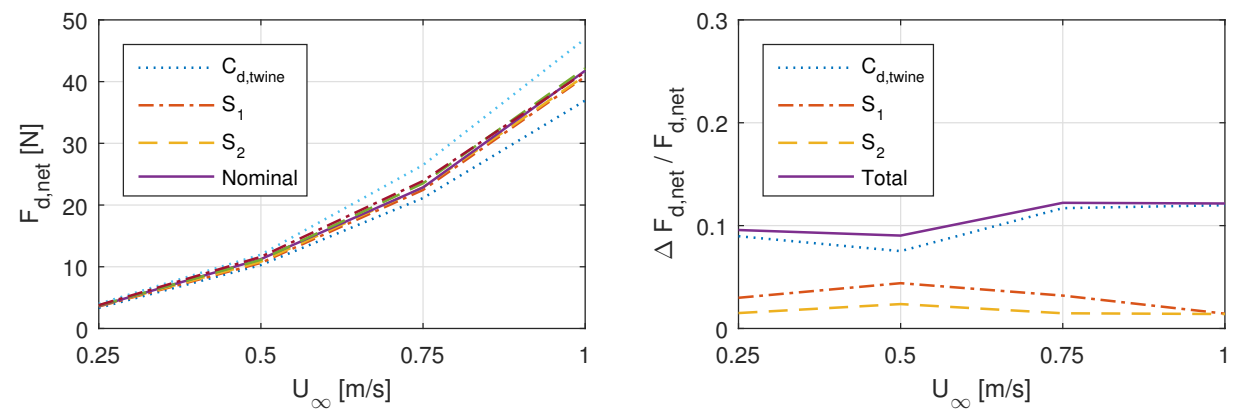

b
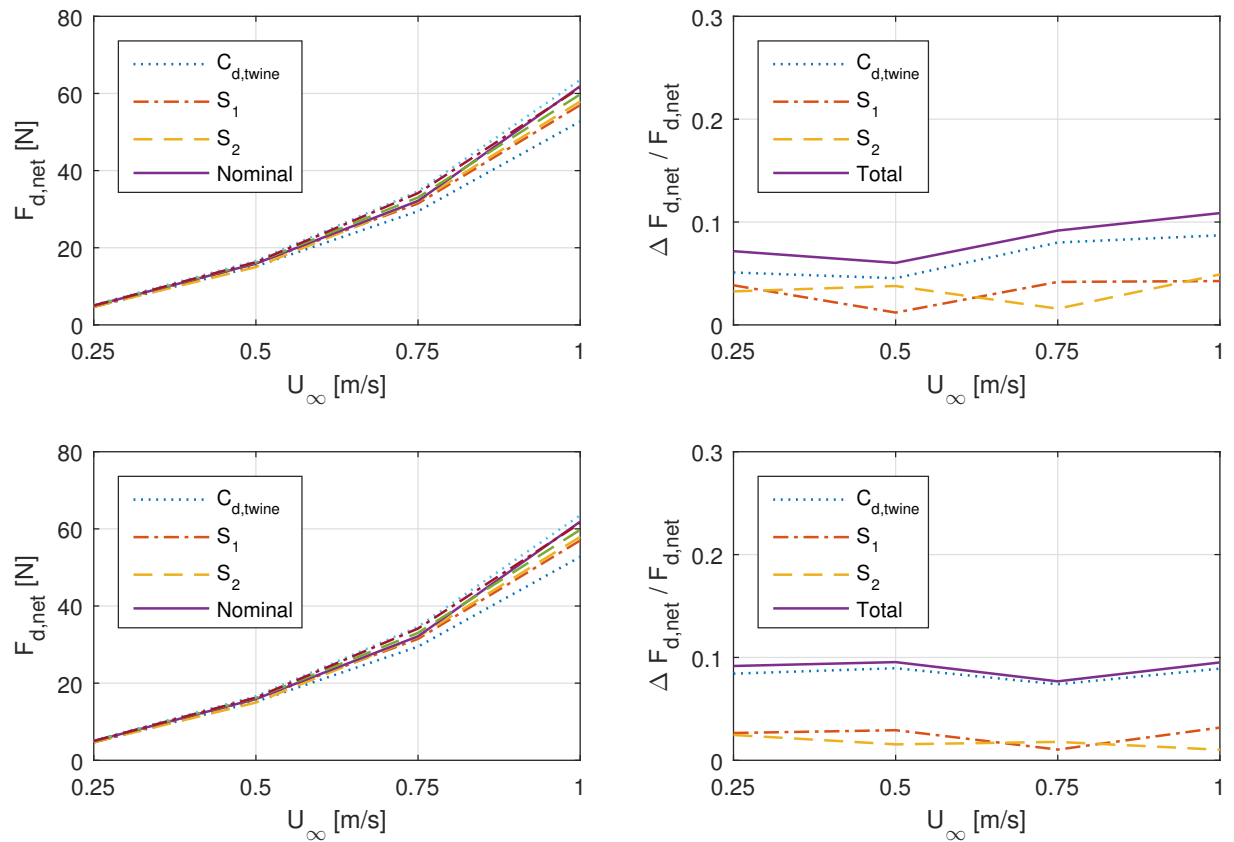

Figure 23: Sensitivity analysis for flow through circular fish cages with different solidity ratios: (a) $S_{n}=0.128$. (b) $S_{n}=0.215$. (c) $S_{n}=0.223$. The left figures shows the error bounds of the drag force of the cages due to numerical model uncertainties: $C_{d, t w i n e}, S_{1}$ and $S_{2}$. The right figures depict the relative error due to these uncertainties.

where

$$
R=\left[\begin{array}{lll}
\cos \left(x, x^{*}\right) & \cos \left(x, y^{*}\right) & \cos \left(x, z^{*}\right) \\
\cos \left(y, x^{*}\right) & \cos \left(y, y^{*}\right) & \cos \left(y, z^{*}\right) \\
\cos \left(z, x^{*}\right) & \cos \left(z, y^{*}\right) & \cos \left(z, z^{*}\right)
\end{array}\right]
$$

where $\cos \left(x_{i}, x_{i}^{*}\right)$ is the cosines of the angle between $x_{i}$ axis and $x_{i}^{*}$ axis where $i=(x, y, z)$. In $2 \mathrm{D}$ cases where $z^{*}$ and $z$ are aligned, we introduce $\zeta$ as the rotation angle from local to global coordinate (the positive direction is based on the right-hand rule). Hereby the transformation matrix was simplified as:

$$
R_{i j}=\left[\begin{array}{cc}
\cos \zeta & \sin \zeta \\
-\sin \zeta & \cos \zeta
\end{array}\right]
$$

This matrix is equivalent to the transformation matrix adopted in Patursson et al. (2010). Substituting Eq. A.3 into Eq. A.1 gave the final expressions of $D$ and $C$ :

$$
D_{i j}=\left[\begin{array}{cc}
D_{1}^{*} \cos ^{2}(\zeta)+D_{2}^{*} \sin ^{2}(\zeta) & -D_{1}^{*} \sin (\zeta) \cos (\zeta)+D_{2}^{*} \cos (\zeta) \sin (\zeta) \\
-D_{1}^{*} \sin (\zeta) \cos (\zeta)+D_{2}^{*} \cos (\zeta) \sin (\zeta) & D_{1}^{*} \sin ^{2}(\zeta)+D_{2}^{*} \cos ^{2}(\zeta)
\end{array}\right]
$$




$$
C_{i j}=\left[\begin{array}{cc}
C_{1}^{*} \cos ^{2}(\zeta)+C_{2}^{*} \sin ^{2}(\zeta) & -C_{1}^{*} \sin (\zeta) \cos (\zeta)+C_{2}^{*} \cos (\zeta) \sin (\zeta) \\
-C_{1}^{*} \sin (\zeta) \cos (\zeta)+C_{2}^{*} \cos (\zeta) \sin (\zeta) & C_{1}^{*} \sin ^{2}(\zeta)+C_{2}^{*} \cos ^{2}(\zeta)
\end{array}\right]
$$

Instead to use matrix multiplication, Zhao et al. (2013b) employed a simplified method as:

$$
\begin{aligned}
& D_{1}=\frac{D_{1}^{*}+D_{2}^{*}}{2}+\frac{D_{1}^{*}-D_{2}^{*}}{2} \cos (2 \zeta)=D_{1}^{*} \cos ^{2} \zeta+D_{2}^{*} \sin ^{2} \zeta \\
& C_{1}=\frac{C_{1}^{*}+C_{2}^{*}}{2}+\frac{C_{1}^{*}-C_{2}^{*}}{2} \cos (2 \zeta)=C_{1}^{*} \cos ^{2} \zeta+C_{2}^{*} \sin ^{2} \zeta \\
& D_{2}=\frac{D_{1}^{*}-D_{2}^{*}}{2} \sin (-2 \zeta)=-D_{1}^{*} \sin \zeta \cos \zeta+D_{2}^{*} \sin \zeta \cos \zeta \\
& C_{2}=\frac{C_{1}^{*}-C_{2}^{*}}{2} \sin (-2 \zeta)=-C_{1}^{*} \sin \zeta \cos \zeta+C_{2}^{*} \sin \zeta \cos \zeta
\end{aligned}
$$

By comparing Eq. A.4- A.5 with Eq. A.6- A.9), it was found that in the transformed $D$ matrix, $D_{11}=D_{1}$ but $D_{12}=D_{21}=D_{2}$, and $D_{22}$ was not calculated in Zhao et al. (2013b). It was the same with $C$. The formulation of porous resistance in Zhao et al. (2013b) should be expressed as:

$$
S=\left[\begin{array}{l}
D_{1} \mu|u|+\frac{1}{2} C_{1} \rho u^{2} \\
D_{2} \mu|u|+\frac{1}{2} C_{2} \rho u^{2}
\end{array}\right]
$$

Therefore the simplification in Zhao et al. (2013b) limits its application, and it is valid only when the flow is unidirectional and the flow direction is aligned with $x$ axis. For wave interaction with fishing nets, Eq. A.6 - A.9 are not valid anymore, and Eq. A.4 A.5 should be used due to the orbital motion of water particles.

\section{Appendix B. The force on porous media based on linear momentum conservation of control volumes}

The forces acting on porous media could be obtained by linear momentum conservation on control volume, as shown in Patursson (2008). Below a detailed derivation is presented for this method.

Assume that the porous media is enclosed by the control volume $C V$. Therefore the integral form of the momentum conservation in the control volume was expressed as:

$$
\frac{\mathrm{d}}{\mathrm{d} t}\left(\int_{C V} \rho u_{i} \mathrm{~d} V\right)=-\int_{C S} p \mathrm{n}_{i} \mathrm{~d} S+\int_{C S} \tau_{i j} \mathrm{n}_{j} \mathrm{~d} S+\int_{C V} \rho g_{i} \mathrm{~d} V-Q_{i}
$$

Here the term in the left hand side in Eq. (B.1) is the material derivative of the momentum on the control volume. The terms on the right-hand side are pressure, viscous stress, body force and finally the force on porous media due to fluid. Note that the force on porous media from the fluid and the force on the fluid from porous media are a pair of force according to Newton's third law.

Furthermore, assume that the control volume is not varying with time, e.g. the porous media is always enclosed by a fixed control volume. Then the volume integral is not a function of time. Then the material derivative of the momentum was given as:

$$
\frac{\mathrm{d}}{\mathrm{d} t}\left(\int_{C V} \rho u_{i} \mathrm{~d} V\right)=\frac{\partial}{\partial t}\left(\int_{C V} \rho u_{i} \mathrm{~d} V\right)+\int_{C V} \frac{\partial}{\partial x_{j}}\left(\rho u_{i} u_{j}\right) \mathrm{d} V
$$

By applying Gauss theorem, the second term on the right-hand side of Eq. (B.2) was converted to surface integral, then Eq. (B.2) was rewritten as:

$$
\frac{\mathrm{d}}{\mathrm{d} t}\left(\int_{C V} \rho u_{i} \mathrm{~d} V\right)=\frac{\partial}{\partial t}\left(\int_{C V} \rho u_{i} \mathrm{~d} V\right)+\int_{C S} \rho u_{i} u_{j} \mathrm{n}_{j} \mathrm{~d} S
$$


Substituting Eq. (B.3) into Eq. (B.1) yields the final expression of $Q_{i}$ :

$$
Q_{i}=-\frac{\partial}{\partial t}\left(\int_{C V} \rho u_{i} \mathrm{~d} V\right)-\int_{C S} \rho u_{i} u_{j} \mathrm{n}_{j} \mathrm{~d} S-\int_{C S} p \mathrm{n}_{i} \mathrm{~d} S+\int_{C S} \tau_{i j} \mathrm{n}_{j} \mathrm{~d} S+\int_{C V} \rho g_{i} \mathrm{~d} V
$$

\section{References}

Balash, C., Colbourne, B., Bose, N., Raman-Nair, W., 2009. Aquaculture net drag force and added mass. Aquacultural Engineering 41, 14-21. URL: http://linkinghub.elsevier.com/retrieve/pii/S0144860909000338 doi 10.1016/j.aquaeng. 2009.04 .003

Berberović, E., van Hinsberg, N., Jakirlić, S., Roisman, I., Tropea, C., 2009. Drop impact onto a liquid layer of finite thickness: Dynamics of the cavity evolution. Physical Review E 79, 036306. URL: http://link.aps.org/doi/10.1103/PhysRevE.79. 036306 , doi 10.1103/PhysRevE.79.036306

Bi, C., Zhao, Y., Dong, G., Zheng, Y., Gui, F., 2014a. A numerical analysis on the hydrodynamic characteristics of net cages using coupled fluid-structure interaction model. Aquacultural Engineering 59, 1-12. URL: http://linkinghub.elsevier. com/retrieve/pii/S014486091400003Xhttp://www.sciencedirect.com/science/article/pii/S014486091400003X doi 10. $1016 / \mathrm{j}$. aquaeng. 2014.01 .002

Bi, C.W., Zhao, Y.P., Dong, G.H., Cui, Y., Gui, F.K., 2015. Experimental and numerical investigation on the damping effect of net cages in waves. Journal of Fluids and Structures 55, 122-138. URL: http://linkinghub.elsevier.com/retrieve/ pii/S0889974615000523 doi 10.1016/j.jfluidstructs.2015.02.010.

Bi, C.W., Zhao, Y.P., Dong, G.H., Xu, T.J., Gui, F.K., 2013a. Experimental investigation of the reduction in flow velocity downstream from a fishing net. Aquacultural Engineering 57, 71-81. URL: http://linkinghub.elsevier.com/retrieve/ pii/S0144860913000691 doi 10.1016/j.aquaeng.2013.08.002

Bi, C.W., Zhao, Y.P., Dong, G.H., Xu, T.J., Gui, F.K., 2013b. Experimental investigation of the reduction in flow velocity downstream from a fishing net. Aquacultural Engineering 57, 71-81. URL: http://linkinghub.elsevier.com/retrieve/ pii/S0144860913000691 doi 10.1016/j.aquaeng.2013.08.002

Bi, C.W., Zhao, Y.P., Dong, G.H., Xu, T.J., Gui, F.K., 2014b. Numerical simulation of the interaction between flow and flexible nets. Journal of Fluids and Structures 45, 180-201. URL: http://linkinghub.elsevier.com/retrieve/pii/ S0889974613002594 doi $10.1016 / \mathrm{j} . j$ fluidstructs.2013.11.015

Bouhoubeiny, E., Germain, G., Druault, P., 2011. Time-Resolved PIV investigations of the flow field around cod-end net structures. Fisheries Research 108, 344-355. URL: http://dx.doi.org/10.1016/j.fishres.2011.01.010 doi $10.1016 / \mathrm{j}$. fishres.2011.01.010

Burcharth, H., Andersen, O., 1995. On the one dimensional steady and unsteady porous flow equations. Coastal Engineering , 233-257.

Darcy, H., 1856. Les fontaines publiques de la ville de Dijon. Dalmont.

Devilliers, M., Vincent, B., Mnassri, I., 2016. A new adaptive mesh refinement to model water flow around fishing nets. Ocean Engineering 113, 34-43. URL: http://www.sciencedirect.com/science/article/pii/S0029801815006630, doi 10.1016/j. oceaneng. 2015.12.009

Fenton, J., 1988. The numerical solution of steady water wave problems. Computers \& Geosciences 14, 357-368. doi 10.1016/ 0098-3004(88) 90066-0.

Forchheimer, H., 1901. Wasserbewegung durch Boden. 45 ed., Zeitschrift des Vereines Deutscher Ingenieure.

van Gent, M.R., 1995. Wave interaction with permeable coastal structures. Ph.d. thesis. Delft University of Technology.

Gopala, V.R., van Wachem, B.G., 2008. Volume of fluid methods for immiscible-fluid and free-surface flows. Chemical Engineering Journal 141, 204-221. URL: http://linkinghub.elsevier.com/retrieve/pii/S1385894708000028 doi 10.1016/j. cej.2007.12.035

Higuera, P., Lara, J.L., Losada, I.J., 2014a. Three-dimensional interaction of waves and porous coastal structures using OpenFOAM. Part I: Formulation and validation. Coastal Engineering 83, 243-258. URL: http://linkinghub.elsevier. com/retrieve/pii/S0378383913001452 doi $10.1016 / \mathrm{j}$. coastaleng.2013.08.010.

Higuera, P., Lara, J.L., Losada, I.J., 2014b. Three-dimensional interaction of waves and porous coastal structures using OpenFOAM. Part II: Application. Coastal Engineering 83, 259-270. URL: http://linkinghub.elsevier.com/retrieve/ pii/S0378383913001464 doi 10.1016/j.coastaleng.2013.09.002.

Hirt, C., Nichols, B., 1981. Volume of fluid (VOF) method for the dynamics of free boundaries. Journal of Computational Physics 39, 201-225. URL: http://www.sciencedirect.com/science/article/pii/0021999181901455, doi 10.1016/0021-9991(81) 90145-5

Hoerner, S., 1965. Fluid-dynamic drag: practical information on aerodynamic drag and hydrodynamic resistance. Hoerner Fluid Dynamics.

Hsu, T.J., Sakakiyama, T., Liu, P.L.F., 2002. A numerical model for wave motions and turbulence flows in front of a composite breakwater. Coastal Engineering 46, 25-50. URL: http://linkinghub.elsevier.com/retrieve/pii/S0378383902000455. doi $10.1016 / \mathrm{S} 0378-3839(02) 00045-5$

Huang, C.C., Tang, H.J., Liu, J.Y., 2006. Dynamical analysis of net cage structures for marine aquaculture: Numerical simulation and model testing. Aquacultural Engineering 35, 258-270. URL: http://linkinghub.elsevier.com/retrieve/ pii/S014486090600029X doi 10.1016/j.aquaeng.2006.03.003.

Jacobsen, N.G., Fuhrman, D.R., Fredsoe, J., 2012. A wave generation toolbox for the open-source CFD library: OpenFoam (R). INTERNATIONAL JOURNAL FOR NUMERICAL METHODS IN FLUIDS 70, 1073-1088. doi 10.1002/fld.2726 
Jacobsen, N.G., van Gent, M.R., Wolters, G., 2015. Numerical analysis of the interaction of irregular waves with two dimensional permeable coastal structures. Coastal Engineering 102, 13-29. URL: http://linkinghub.elsevier.com/retrieve/pii/ S0378383915000836 doi $10.1016 / \mathrm{j}$.coastaleng.2015.05.004

Jensen, B., Jacobsen, N.G.l., Christensen, E.D., 2014. Investigations on the porous media equations and resistance coefficients for coastal structures. Coastal Engineering 84, 56-72. URL: http://linkinghub.elsevier.com/retrieve/pii/ S0378383913001816 doi $10.1016 / j$.coastaleng.2013.11.004

del Jesus, M., 2011. Three-dimensional Interaction of Water Waves with Maritime Structures. Ph.d. thesis. University of Cantabria.

del Jesus, M., Lara, J.L., Losada, I.J., 2012. Three-dimensional interaction of waves and porous coastal structures. Coastal Engineering 64, 57-72. URL: http://linkinghub.elsevier.com/retrieve/pii/S0378383912000245 doi 10.1016/j.coastaleng. 2012.01 .008

Kim, Y.H., 2012. Analysis of turbulence and tilt by in-situ measurements inside the codend of a shrimp beam trawl. Ocean Engineering 53, 6-15. URL: http://dx.doi.org/10.1016/j.oceaneng.2012.06.014, doi:10.1016/j.oceaneng.2012.06.014

Kristiansen, T., Faltinsen, O.M., 2012. Modelling of current loads on aquaculture net cages. Journal of Fluids and Structures 34, 218-235. URL: http://linkinghub.elsevier.com/retrieve/pii/S0889974612000783, doi 10.1016/j.jfluidstructs . 2012.04 .001

Kristiansen, T., Faltinsen, O.M., 2015. Experimental and numerical study of an aquaculture net cage with floater in waves and current. Journal of Fluids and Structures 54, 1-26. URL: http://linkinghub.elsevier.com/retrieve/pii/ S0889974614002114 doi $10.1016 / \mathrm{j} \cdot \mathrm{jfluidstructs.2014.08.015}$

Lader, P.l., Fredriksson, D.W., Guenther, J., Volent, Z., Blocher, N., Kristiansen, D., Gansel, L., Decew, J., 2015. Drag on hydroid-fouled nets An experimental approach. China Ocean Engineering 29, 369-389. URL: http://link.springer.com/ 10.1007/s13344-015-0026-y doi 10.1007/s13344-015-0026-y

Lader, P.l., Jensen, A., Sveen, J.K., Fredheim, A., Enerhaug, B., Fredriksson, D., 2007a. Experimental investigation of wave forces on net structures. Applied Ocean Research 29, 112-127. URL: http://linkinghub.elsevier.com/retrieve/pii/ S0141118707000697 doi $10.1016 / \mathrm{j}$. apor.2007.10.003

Lader, P.l.F., Olsen, A., Jensen, A., Sveen, J.K., Fredheim, A., Enerhaug, B., 2007b. Experimental investigation of the interaction between waves and net structures-damping mechanism. Aquacultural Engineering 37, 100-114. URL: http: //linkinghub.elsevier.com/retrieve/pii/S0144860907000362 doi 10.1016/j.aquaeng.2007.03.001

Liu, P.L.F., Lin, P., Chang, K.A., Sakakiyama, T., 1999. Numerical modeling of wave interaction with porous structures. Journal of Waterway, Port, Coastal, and Ocean Engineering 125, 322-330. doi 10.1061/(ASCE) 0733-950X (2001) 127:2(123)

Løland, G., 1993. Current forces on, and water flow through and around, floating fish farms. Aquaculture International 1, 72-89. doi 10.1007/BF00692665

Losada, I.J., Lara, J.L., Guanche, R., Gonzalez-Ondina, J.M., 2008. Numerical analysis of wave overtopping of rubble mound breakwaters. Coastal Engineering 55, 47-62. doi 10.1016/j.coastaleng.2007.06.003

Márquez Damián, S., 2013. An Extended Mixture Model for the Simultaneous Treatment of Short and Long Scale Interfaces. Ph.d. thesis. Universidad Nacional del Litoral.

Moe, H., Fredheim, a., Hopperstad, O.S., 2010. Structural analysis of aquaculture net cages in current. Journal of Fluids and Structures 26, 503-516. URL: http://linkinghub.elsevier.com/retrieve/pii/S0889974610000289, doi/10.1016/j. jfluidstructs.2010.01.007.

Nakayama, A., Kuwahara, F., 1999. A Macroscopic Turbulence Model for Flow in a Porous Medium. Journal of Fluids Engineering 121, 427-433.

Patursson, O., 2007. Measurements of drag and lift forces on a net panel and the current reduction in the wake of the panel. Technical Report NVDRit2007:10. University of the Faroe Island. Torshavn, Faro Islands.

Patursson, O., 2008. Flow through and around fish farming nets. Ph.d. thesis. University of New Hampshire.

Patursson, O., Swift, M.R., Tsukrov, I., Simonsen, K., Baldwin, K., Fredriksson, D.W., Celikkol, B., 2010. Development of a porous media model with application to flow through and around a net panel. Ocean Engineering 37, 314-324. URL: http://linkinghub.elsevier.com/retrieve/pii/S0029801809002406 doi $10.1016 / j .0 c e a n e n g .2009 .10 .001$

Pichot, G., Germain, G., Priour, D., 2009. On the experimental study of the flow around a fishing net. European Journal of Mechanics, B/Fluids 28, 103-116. URL: http://dx.doi.org/10.1016/j.euromechflu.2008.02.002 doi 10.1016/j. euromechflu.2008.02.002

Rudi, H., L $\varnothing$ land, G., Furunes, I., 1988. Experiments with nets; forces on and flow through net panels and cage systems. Technical Report MT51 F88-0215. MARINTEK. Trondheim, Norway.

Schlichting, H., Gersten, K., 2003. Boundary-Layer Theory. Springer Berlin Heidelberg.

Sumer, B., Fredsøe, J., 2006. Hydrodynamics Around Cylindrical Strucures. Advanced series on ocean engineering, World Scientific Publishing. URL: https://books.google.dk/books?id=-lbnuyzAXOkC.

Wroniszewski, P.a., Verschaeve, J.C., Pedersen, G.K., 2014. Benchmarking of NavierStokes codes for free surface simulations by means of a solitary wave. Coastal Engineering 91, 1-17. URL: http://linkinghub.elsevier.com/retrieve/pii/ S0378383914000842 doi $10.1016 / \mathrm{j}$.coastaleng.2014.04.012

Xu, T.J., Zhao, Y.P., Dong, G.H., Gui, F.K., 2013a. Analysis of hydrodynamic behavior of a submersible net cage and mooring system in waves and current. Applied Ocean Research 42, 155-167. URL: http://dx.doi.org/10.1016/j.apor.2013.05.007. doi $10.1016 /$ j.apor.2013.05.007.

Xu, T.J., Zhao, Y.P., Dong, G.H., Li, Y.C., Gui, F.K., 2013b. Analysis of hydrodynamic behaviors of multiple net cages in combined wavecurrent flow. Journal of Fluids and Structures 39, 222-236. URL: http://linkinghub.elsevier.com/ retrieve/pii/S088997461300042X doi $10.1016 / \mathrm{j} \cdot \mathrm{jfluidstructs.2013.02.011}$

Zhan, J., Jia, X., Li, Y., Sun, M., Guo, G., Hu, Y., 2006. Analytical and experimental investigation of drag on nets of fish 
cages. Aquacultural Engineering 35, 91-101. URL: http://linkinghub.elsevier.com/retrieve/pii/S0144860905001275 doi $10.1016 / j$. aquaeng. 2005.08.013

Zhao, Y.P., Bi, C.W., Dong, G.H., Gui, F.K., Cui, Y., Guan, C.T., Xu, T.J., 2013a. Numerical simulation of the flow around fishing plane nets using the porous media model. Ocean Engineering 62, 25-37. URL: http://linkinghub.elsevier.com/ retrieve/pii/S0029801813000243 doi 10.1016/j.oceaneng.2013.01.009.

Zhao, Y.P., Bi, C.W., Dong, G.H., Gui, F.K., Cui, Y., Xu, T.J., 2013b. Numerical simulation of the flow field inside and around gravity cages. Aquacultural Engineering 52, 1-13. URL: http://linkinghub.elsevier.com/retrieve/pii/ S0144860912000593 doi $10.1016 / j$.aquaeng.2012.06.001

Zhao, Y.p., Bi, C.w., Liu, Y.x., Dong, G.h., Gui, F.k., Science, M., 2014. Numerical simulation of interaction between waves and net panel using porous media model. Engineering Applications of Computational Fluid Mechanics 8, 116-126. 